\title{
High scale validity of the DFSZ axion model with precision
}

\author{
Satsuki Oda, ${ }^{a, b}$ Yutaro Shoji ${ }^{c}$ and Dai-suke Takahashi ${ }^{a, b}$ \\ ${ }^{a}$ Okinawa Institute of Science and Technology Graduate University (OIST), \\ Onna, Okinawa 904-0495, Japan \\ ${ }^{b}$ Institute for Pacific Rim Studies, Meio University, \\ Nago, Okinawa 905-8585, Japan \\ ${ }^{c}$ Kobayashi-Maskawa Institute for the Origin of Particles and the Universe, \\ Nagoya University, \\ Nagoya, Aichi 464-8602, Japan \\ E-mail: satsuki.oda@oist.jp, yshoji@kmi.nagoya-u.ac.jp, \\ daisuke.takahashi@oist.jp
}

ABSTRACT: With the assumption of classical scale invariance at the Planck scale, the DFSZ axion model can generate the Higgs mass terms of the appropriate size through technically natural parameters and may be valid up to the Planck scale. We discuss the high scale validity of the Higgs sector, namely the absence of Landau poles and the vacuum stability. The Higgs sector is identical to that of the type-II two Higgs doublet model with a limited number of the Higgs quartic couplings. We utilize the state-of-the-art method to calculate vacuum decay rates and find that they are enhanced at most by $10^{10}$ compared with the tree level evaluation. We also discuss the constraints from flavor observables, perturbative unitarity, oblique parameters and collider searches. We find that the high scale validity tightly constrains the parameter region, but there is still a chance to observe at most about $10 \%$ deviation of the $125 \mathrm{GeV}$ Higgs couplings to the fermions.

Keywords: Beyond Standard Model, Higgs Physics

ArXiv EPrint: 1912.01147 


\section{Contents}

1 Introduction 1

2 DFSZ axion model 3

3 Vacuum decay rate $\quad 5$

3.1 Formulation 5

3.2 Example 8

4 Low energy constraints $\quad 9$

$\begin{array}{lll}4.1 & \text { Flavor observables } & 10\end{array}$

$\begin{array}{lll}4.2 & \text { Low energy perturbative unitarity } & 10\end{array}$

$\begin{array}{lll}4.3 & \text { Oblique parameters } & 12\end{array}$

$\begin{array}{lll}4.4 & \text { Collider searches } & 12\end{array}$

5 High scale validity 12

6 Summary 17

$\begin{array}{lr}\text { A Flavor } & 17\end{array}$

$\begin{array}{ll}\text { A.1 CKM matrix elements } & 17\end{array}$

$\begin{array}{lll}\text { A.2 } & \text { Flavor constraints } & 19\end{array}$

B Proof of straight bounce $\quad 20$

$\begin{array}{ll}\text { C One-loop corrections to a vacuum decay rate } & 21\end{array}$

D Matching conditions 23

D.1 Gauge couplings 23

D.2 Yukawa couplings 24

D.3 Higgs quartic couplings 25

E Generation of data points $\quad 26$

E.1 Necessary conditions for perturbative unitarity 26

$\begin{array}{lll}\text { E.2 Data generation } & 27\end{array}$

$\begin{array}{ll}\text { F Couplings and partial decay widths of heavy higgs bosons } & 29\end{array}$

\section{Introduction}

An invisible axion [1-11] is one of the plausible solutions to the strong CP problem and is also an excellent dark matter candidate. We focus on the DFSZ axion model [10, 11], where the standard model (SM) is extended with a SM singlet complex scalar and an additional Higgs doublet. Since the Higgs doublets have non-zero Peccei-Quinn (PQ) charges, the Higgs couplings are tightly restricted by the PQ symmetry. For example, dangerous flavor 
changing neutral currents (FCNC) are forbidden at the tree level and the $\mathrm{CP}$ is not broken spontaneously in the scalar sector.

In this paper, we discuss the possibility that the DFSZ axion model remains valid up to the Planck scale. In such a scenario, one of the disadvantages is that we need to give up a complete explanation of the hierarchy between the Planck scale and the electroweak (EW) scale. However, if there is a mechanism that realizes classical scale invariance at the Planck scale, the hierarchy problem may be solved without introducing supersymmetry or compositeness [12-14]. Since the scale invariance is violated at the quantum level, the PQ breaking scale can appear through the dimensional transmutation. If the PQ sector and the Higgs sector are connected by (technically natural) tiny couplings, the PQ breaking can also generate the Higgs mass terms without causing a hierarchy problem $[15,16]$. Since the PQ breaking sector decouples from the Higgs sector due to the tiny couplings, the model is well approximated by the type-II two Higgs doublet model (THDM) with a restricted number of coupling constants. Importantly, the additional Higgs bosons should be around the EW scale in this scenario since there is no technically natural parameter that accommodates a hierarchy among the Higgs boson masses.

Another disadvantage is that the model does not explain the neutrino masses, the baryon asymmetry of the Universe, or inflation. However, they can be explained without affecting the Higgs sector. For example, one may consider the see-saw mechanism with right handed neutrinos having a few orders of magnitude smaller masses than the PQ breaking scale [17-19]. It can explain the neutrino masses and also the baryon asymmetry of the Universe with $\tan \beta \gtrsim 4$ [20]. However, it does not cause the hierarchy problem thanks to the tiny Yukawa couplings of the right handed neutrinos. As for inflation, one may attach an inflation sector to the model and assume tiny couplings between the inflation sector and the Higgs sector, which is, at least, technically natural.

For the model to be valid up to the Planck scale, Landau poles should not appear during the renormalization group (RG) evolution and the lifetime of the EW vacuum should be long enough. We refer to these two conditions as the high scale validity. Similar discussions can be found in the context of THDMs [21-33]. As we will see and as found in the previous studies, these conditions are complementary and become very restrictive if combined. Thus, the model becomes more predictive and it is important to determine the allowed parameter space precisely.

The lifetime of the EW vacuum is estimated by the bubble nucleation rate [34, 35], which has a form of

$$
\gamma=\mathcal{A} e^{-\mathcal{B}}
$$

where $\mathcal{B}$ is the Euclidean action of the so-called the bounce, and $\mathcal{A}$ represents quantum corrections to $\mathcal{B}$ having mass dimension four. In many papers, $\mathcal{A}$ is assumed to lie around the typical scale of the problem, but it has been pointed out [36] that such an estimation leads to theoretical uncertainty of $e^{-\mathcal{B} \times \mathcal{O}(10 \%)}$ in the nucleation rate. As we will see later, it can become comparable with the uncertainties coming from those of the top mass and the strong coupling. Thus, it is important to calculate both of $\mathcal{A}$ and $\mathcal{B}$ to get a precise vacuum decay rate. 
The one-loop calculation of $\mathcal{A}$ for the SM was first calculated in [37]. Since the treatment of the gauge zero mode had not been known at that time, the calculation was not complete. Recently, the correct treatment has been found [38] and the one-loop calculation for the SM has been completed [39-41]. In addition, the analytic expression for $\mathcal{A}$ at the one-loop level has become available $[39,41]$ for an approximately scale invariant theory. Since they are applicable to the case where the bounce is composed of a single field, we extend them to a multi-field case in this paper. Differently from the single-field case, there can be more than one unstable directions and there can appear an additional zero mode due to a global symmetry breaking. In addition, the electromagnetic U(1) symmetry can also be broken spontaneously.

Before the analysis of the high scale validity, we impose the constraints from flavor observables, perturbative unitarity, oblique parameters and collider searches. For the constraints from flavor observables, we obtain the $95 \%$ exclusion limit in appendix A using the recent experimental values.

We determine the allowed parameter space by utilizing the Monte Carlo method. We show how much the high scale validity narrows down the parameter space and discuss the implications on the Higgs couplings and the Higgs mass splittings.

This paper is organized as follows. In section 2, we briefly explain the DFSZ axion model. Section 3 is devoted to the details of the analysis on the bubble nucleation rate for the multi-field case. Then, in section 4, we discuss the low energy constraints. In section 5, we execute numerical analysis and discuss the consequence of the high scale validity. Finally, we summarize in section 6.

\section{DFSZ axion model}

In this section, we briefly review the DFSZ axion model [10, 11]. The scalar sector consists of two Higgs doublets, $H_{1}$ and $H_{2}$, and a SM singlet complex scalar, $\Phi$. We choose the PQ charges of $H_{1}, H_{2}$ and $\Phi$ to be $x_{1}, x_{2}$ and $\left(x_{2}-x_{1}\right) / 2$, respectively. Here, we assume $x_{1} \neq x_{2}$ so that $\Phi$ has a non-zero PQ charge.

The general scalar potential is given by

$$
\begin{aligned}
V\left(H_{1}, H_{2}, \Phi\right)= & \tilde{m}_{1}^{2} H_{1}^{\dagger} H_{1}+\tilde{m}_{2}^{2} H_{2}^{\dagger} H_{2}+\frac{\lambda_{1}}{2}\left(H_{1}^{\dagger} H_{1}\right)^{2}+\frac{\lambda_{2}}{2}\left(H_{2}^{\dagger} H_{2}\right)^{2} \\
& +\lambda_{3}\left(H_{1}^{\dagger} H_{1}\right)\left(H_{2}^{\dagger} H_{2}\right)+\lambda_{4}\left(H_{1}^{\dagger} H_{2}\right)\left(H_{2}^{\dagger} H_{1}\right) \\
& +\tilde{\lambda}_{\Phi}\left(|\Phi|^{2}-v_{\Phi}^{2}\right)^{2} \\
& +|\Phi|^{2}\left(\tilde{\kappa}_{1} H_{1}^{\dagger} H_{1}+\tilde{\kappa}_{2} H_{2}^{\dagger} H_{2}\right)-\left(\tilde{\kappa}_{3} \Phi^{2} H_{2}^{\dagger} H_{1}+\text { h.c. }\right),
\end{aligned}
$$

where $v_{\Phi}^{2}, \tilde{m}_{i}^{2}$ 's, $\lambda_{i}{ }^{\prime}$ s, $\tilde{\lambda}_{\Phi}$ and $\tilde{\kappa}_{i}$ 's are constants. We assume $\tilde{\lambda}_{\Phi}$ is moderate so that the VEV of $\Phi$ is not affected by those of $H_{1}$ and $H_{2}$.

We assume the classical scale invariance and set $\tilde{m}_{1}^{2}$ and $\tilde{m}_{2}^{2}$ to zero at the Planck scale. Then, the Higgs mass terms are assumed to be generated through the PQ symmetry breaking. In order to obtain the EW scale, $\tilde{\kappa}_{i}$ 's should be very small since $\Phi$ has to develop a huge vacuum expectation value (VEV) to avoid the constraints on the axion 


\begin{tabular}{|cccccccc|}
\hline \multicolumn{10}{|c|}{ PQ Charge Assignment } \\
\hline$H_{1}$ & $H_{2}$ & $\Phi$ & $Q$ & $L$ & $U$ & $D$ & $E$ \\
\hline$x_{1}$ & $x_{2}$ & $\frac{x_{2}-x_{1}}{2}$ & 0 & 0 & $x_{2}$ & $-x_{1}$ & $-x_{1}$ \\
\hline
\end{tabular}

Table 1. Assignment of the PQ charge in the DFSZ axion model.

decay constant, $10^{9} \mathrm{GeV} \lesssim f_{a} \lesssim 10^{12} \mathrm{GeV}[42,43]$. Due to the smallness of $\tilde{\kappa}_{i}$ 's, $\Phi$ decouples from the Higgs sector and the potential reduces to

$$
\begin{aligned}
V_{\mathrm{THDM}}= & m_{1}^{2} H_{1}^{\dagger} H_{1}+m_{2}^{2} H_{2}^{\dagger} H_{2}-\left(m_{3}^{2} H_{2}^{\dagger} H_{1}+\text { h.c. }\right)+\frac{\lambda_{1}}{2}\left(H_{1}^{\dagger} H_{1}\right)^{2} \\
& +\frac{\lambda_{2}}{2}\left(H_{2}^{\dagger} H_{2}\right)^{2}+\lambda_{3}\left(H_{1}^{\dagger} H_{1}\right)\left(H_{2}^{\dagger} H_{2}\right)+\lambda_{4}\left(H_{1}^{\dagger} H_{2}\right)\left(H_{2}^{\dagger} H_{1}\right),
\end{aligned}
$$

where

$$
\begin{aligned}
& m_{1}^{2}=\tilde{\kappa}_{1} v_{\Phi}^{2}, \\
& m_{2}^{2}=\tilde{\kappa}_{2} v_{\Phi}^{2}, \\
& m_{3}^{2}=\left|\tilde{\kappa}_{3}\right| v_{\Phi}^{2} .
\end{aligned}
$$

Here, we took $m_{3}^{2}$ to be real and positive by the redefinition of the phase of $H_{1}$. Notice that PQ violating quartic couplings can be generated after the PQ symmetry breaking, but they are suppressed by $\tilde{\kappa}_{i}$ 's and hence are negligible.

With the PQ charge assignment shown in table 1, the Higgs doublets couple to the $\mathrm{SM}$ fermions as

$$
\mathcal{L}_{\text {Yukawa }}=-y_{U} \bar{Q} \tilde{H}_{2} U-y_{D} \bar{Q} H_{1} D-y_{E} \bar{L} H_{1} E+\text { h.c. },
$$

with

$$
\tilde{H}_{2}=i \sigma_{2} H_{2}^{*} \text {. }
$$

Here, $i \sigma_{2}$ is the completely anti-symmetric matrix and $Q, L, U, D$ and $E$ represent the left quark doublets, the left lepton doublets, the up-type quarks, the down-type quarks and the charged leptons in the SM, respectively. The model is thus regarded as the type-II THDM with a limited number of Higgs quartic couplings.

Let us define the mass eigenstates and the mixing angles. We expand the Higgs fields as

$$
H_{j}=\left(\begin{array}{c}
\omega_{j}^{+} \\
\left(v_{j}+h_{j}-i \zeta_{j}\right) / \sqrt{2}
\end{array}\right)
$$

with

$$
\left(\begin{array}{l}
h_{1} \\
h_{2}
\end{array}\right)=R(\alpha)\left(\begin{array}{c}
H \\
h
\end{array}\right),\left(\begin{array}{l}
\zeta_{1} \\
\zeta_{2}
\end{array}\right)=R(\beta)\left(\begin{array}{c}
G^{0} \\
A
\end{array}\right),\left(\begin{array}{c}
\omega_{1}^{+} \\
\omega_{2}^{+}
\end{array}\right)=R(\beta)\left(\begin{array}{c}
G^{+} \\
H^{+}
\end{array}\right),
$$

where $v_{i}$ 's are the VEVs of the Higgs fields, $\tan \beta=v_{2} / v_{1}$ and

$$
R(\theta)=\left(\begin{array}{cc}
\cos \theta & -\sin \theta \\
\sin \theta & \cos \theta
\end{array}\right) \text {. }
$$


Here, $h$ is the $125 \mathrm{GeV}$ Higgs boson, $H$ is the additional CP-even Higgs boson, $A$ is the CP-odd Higgs boson, $H^{+}$is the charged Higgs boson, and $G^{0}$ and $G^{+}$are the would-be Nambu-Goldstone bosons. The SM-like limit for $h$ is given by $\beta-\alpha \rightarrow \pi / 2$.

\section{Vacuum decay rate}

Since the quantum corrections to the effective potential depend on the VEVs of the Higgs fields, the shape of the effective potential is non-trivial at large Higgs VEVs. When there is a deeper vacuum or the effective potential is unbounded from below, the EW vacuum is not absolutely stable and decays through quantum tunneling. Even in such a case, we can live in the meta-stable vacuum if it has a much longer lifetime than the age of the Universe. In this section, we discuss the precise determination of vacuum decay rates for the DFSZ axion model.

\subsection{Formulation}

Recently, the analytic formulas for the prefactor, $\mathcal{A}$, at the one-loop level have been derived $[39,41]$, which are applicable to the case where the theory is approximately scale invariant and the bounce consists of a single field. In the following, we extend their results to the case where the bounce consists of more than one fields.

Since the PQ-breaking sector couples to the THDM sector very weakly, the vacuum decay rate can be calculated independently of the PQ-breaking sector, i.e. the decay path, the RG running or the calculation of $\mathcal{A}$ is not affected by the PQ-breaking sector. ${ }^{1}$ Notice that even when the field value of $H_{1}$ or $H_{2}$ becomes much larger than the PQ-breaking scale, $\Phi$ is almost constant during the tunneling. This is because the typical size of a bounce, i.e. $\bar{R} \simeq 1 / \sqrt{\left|H_{1}(0)\right|^{2}+\left|H_{2}(0)\right|^{2}}$, is too small. Here, $H_{i}(0)$ 's are the field values at the center of the bounce. For example, let us assume that $\Phi$ obtains a negative mass squared, $m_{\Phi}^{2}<0$, during the tunneling. Then, the displacement of $\Phi$ is roughly estimated as $v_{\Phi}\left(e^{\sqrt{\left|m_{\Phi}^{2}\right|} \bar{R}}-1\right)$, which is negligible since $\left|m_{\Phi}^{2}\right| \ll 1 / \bar{R}^{2}$.

Since the field value at the true vacuum is typically much larger than the EW scale, ${ }^{2}$ the Higgs potential is approximately given by

$$
V_{\mathrm{THDM}} \simeq \frac{\lambda_{1}}{2}\left(H_{1}^{\dagger} H_{1}\right)^{2}+\frac{\lambda_{2}}{2}\left(H_{2}^{\dagger} H_{2}\right)^{2}+\lambda_{3}\left(H_{1}^{\dagger} H_{1}\right)\left(H_{2}^{\dagger} H_{2}\right)+\lambda_{4}\left(H_{1}^{\dagger} H_{2}\right)\left(H_{2}^{\dagger} H_{1}\right) .
$$

For the moment, we fix the renormalization scale and will discuss the running effect later.

The bounce is a solution to the Euclidean equations of motion that are given by

$$
\begin{aligned}
& \frac{d^{2} H_{1}^{i}}{d r^{2}}+\frac{3}{r} \frac{d H_{1}^{i}}{d r}=\frac{\partial V_{\mathrm{THDM}}}{\partial H_{1}^{i *}}, \\
& \frac{d^{2} H_{2}^{i}}{d r^{2}}+\frac{3}{r} \frac{d H_{2}^{i}}{d r}=\frac{\partial V_{\mathrm{THDM}}}{\partial H_{2}^{i *}},
\end{aligned}
$$

\footnotetext{
${ }^{1}$ If the potential of the PQ field itself is unstable, we need to calculate the vacuum decay rate in the PQ sector and add it to that in the THDM sector. In this paper, we assume the stable potential of the PQ field given in eq. (2.1).

${ }^{2}$ The another vacuum may be close to the EW vacuum, which happens when the low energy potential already has an instability and the RG running cures it above the EW scale. We will put an IR cut-off on the size of the bounce to avoid such a situation.
} 
with boundary conditions,

$$
\frac{d H_{1}^{i}}{d r}(0)=\frac{d H_{2}^{i}}{d r}(0)=0, H_{1}^{i}(\infty)=H_{2}^{i}(\infty)=0,
$$

and their complex conjugates. Here, $r$ is the radius from the center of the bubble and $i=1,2$ labels the components of the doublet. Without loss of generality, ${ }^{3}$ we parameterize the Higgs fields as

$$
H_{1}=\frac{1}{\sqrt{2}}\left(\begin{array}{c}
0 \\
\phi \cos \Omega
\end{array}\right), \quad H_{2}=\frac{1}{\sqrt{2}} e^{i\left(\sigma_{1} \theta_{1}+\sigma_{2} \theta_{2}\right)} e^{i \sigma_{3} \theta_{3}}\left(\begin{array}{c}
0 \\
\phi \sin \Omega
\end{array}\right),
$$

where $\sigma_{i}$ 's are the Pauli matrices. Then, the potential is expressed as

$$
V_{\mathrm{THDM}} \simeq \frac{\lambda_{\phi}(\Omega, \Theta)}{4} \phi^{4},
$$

where

$$
\begin{aligned}
\lambda_{\phi}(\Omega, \Theta) & =\frac{1}{2}\left[\lambda_{1} \cos ^{4} \Omega+\lambda_{2} \sin ^{4} \Omega+2\left(\lambda_{3}+\lambda_{4} \cos ^{2} \Theta\right) \sin ^{2} \Omega \cos ^{2} \Omega\right], \\
\Theta & =\sqrt{\theta_{1}^{2}+\theta_{2}^{2}} .
\end{aligned}
$$

In appendix $\mathrm{B}$, we show that $\Omega$ and $\Theta$ are constant. ${ }^{4}$ Then, the equations of motion reduce to ${ }^{5}$

$$
\begin{aligned}
\frac{\partial \lambda_{\phi}}{\partial \Theta} & =0, \\
\frac{\partial \lambda_{\phi}}{\partial \Omega} & =0 \\
\frac{d^{2} \phi}{d r^{2}}+\frac{3}{r} \frac{d \phi}{d r} & =\lambda_{\phi} \phi^{3},
\end{aligned}
$$

with boundary conditions

$$
\frac{d \phi}{d r}(0)=0, \phi(\infty)=0 .
$$

From eq. (3.7), we can see that a minimum of $\lambda_{\phi}$ satisfies $\cos ^{2} \Theta=0$ for $\lambda_{4} \geq 0$, and $\cos ^{2} \Theta=1$ for $\lambda_{4}<0$. Then, from eq. (3.10), we get the following solutions;

$$
\begin{aligned}
& \text { (a) } \Omega=0, \quad \lambda_{\phi}=\frac{1}{2} \lambda_{1}, \\
& \text { (b) } \Omega=\frac{\pi}{2}, \quad \lambda_{\phi}=\frac{1}{2} \lambda_{2}, \\
& \text { (c) } \tan ^{2} \Omega=\frac{\lambda_{1}-\bar{\lambda}}{\lambda_{2}-\bar{\lambda}}, \quad \lambda_{\phi}=\frac{1}{2} \frac{\lambda_{1} \lambda_{2}-\bar{\lambda}^{2}}{\lambda_{1}+\lambda_{2}-2 \bar{\lambda}},
\end{aligned}
$$

\footnotetext{
${ }^{3}$ We work in the Fermi gauge as in [41] and we pick up one representative element.

${ }^{4}$ Quantum corrections to the bounce may depend on $\Omega$ or $\Theta$. However, they result in two- or higher-loop corrections to a vacuum decay rate since the bounce is a saddle point of the action.

${ }^{5}$ Since the potential is independent of $\theta_{3}$, there exist an infinite number of bounce solutions and a zero mode appears in the calculation of the functional determinant. We follow [41] for the treatment of the zero mode.
} 
where

$$
\bar{\lambda}=\min \left(\lambda_{3}, \lambda_{3}+\lambda_{4}\right) .
$$

Notice that $(c)$ exists only when $\left(\lambda_{1}-\bar{\lambda}\right) /\left(\lambda_{2}-\bar{\lambda}\right)>0$.

If $\lambda_{\phi}<0$, the solution to eqs. (3.11) and (3.12) is given by

$$
\phi(r)=\sqrt{\frac{8}{\left|\lambda_{\phi}\right|}} \frac{R}{R^{2}+r^{2}}
$$

which gives

$$
\mathcal{B}=\frac{8 \pi^{2}}{3\left|\lambda_{\phi}\right|}
$$

with $R$ being a free parameter that fixes the radius of the bounce. Notice that $\mathcal{B}$ is independent of $R$, which is due to the (approximate) classical scale invariance.

Since all the possible bounces contribute to the vacuum decay rate, the total vacuum decay rate is expressed as

$$
\gamma=\sum_{\lambda_{\phi}} \int d R \frac{d \gamma}{d R}
$$

where $\lambda_{\phi}$ is summed over its minima with $\lambda_{\phi}<0$. Now, the problem is reduced to the single field case for each $\lambda_{\phi}$ and we can use the one-loop results of [39-41]. The details are in appendix $\mathrm{C}$.

Let us discuss the convergence of the $R$ integral. From the dimensional analysis and the renormalization scale independence of the vacuum decay rate, the $R$-dependence of the integrand can be determined as

$$
\frac{d \gamma}{d R} \propto R^{-5}(\mu R)^{-\frac{8 \pi^{2} \beta_{\lambda_{\phi}}^{(1)}}{3 \lambda_{\phi}^{2}}},
$$

at the one-loop level. Here, $\mu$ is the renormalization scale and $\beta_{\lambda_{\phi}}^{(1)}$ is the one-loop beta function for $\lambda_{\phi}$. Thus, if we integrate it over $R \in(0, \infty)$, the integration does not converge. However, as discussed in [39-41], the result can be convergent if we include higher-loop corrections. Although it is very difficult to calculate them, their $R$-dependence is completely determined by the beta functions and we can sum up the logarithmic corrections by taking $\mu \sim 1 / R$ for each bounce with radius $R$ (for detailed discussion see [41]). If there exists a minimum of the effective action, it dominates the $R$ integral and the result is convergent.

Independently of the convergence of the $R$ integral, we use cut-offs for the $R$ integral for the following reasons. First, we need an IR cut-off because we have ignored the dimensionful couplings. ${ }^{6}$ Second, we need a UV cut-off because we do not consider gravitational corrections. Thus, we set the integration region as ${ }^{7}$

$$
\gamma=\sum_{\lambda_{\phi}} \int_{1 / M_{\mathrm{Pl}}}^{1 /(10 \mathrm{TeV})} d R \frac{d \gamma}{d R},
$$

\footnotetext{
${ }^{6}$ The effect of the mass term of the bounce field at the false vacuum, $m^{2}$, is discussed in [39] and is shown to be suppressed by $R^{2} m^{2}$. We will discuss the cut-off dependence later.

${ }^{7}$ We will discuss the cut-off dependence later.
} 
where $M_{\mathrm{Pl}}$ is the reduced Planck scale. We also impose the same limits on the field value of the bounce as

$$
10 \mathrm{TeV} \lesssim \phi(0)=\sqrt{\frac{8}{\left|\lambda_{\phi}(\mu)\right|}} \frac{1}{R} \lesssim M_{\mathrm{Pl}}
$$

when $\lambda_{\phi}<0$.

We also exclude the region where the quantum corrections to the action become larger than $80 \%$ of $\mathcal{B}$ since the perturbative expansions become unreliable. Such a region appears where $\lambda_{\phi}$ is very close to zero.

Since the integrand of the vacuum decay rate is positive definite, these limits always make the vacuum decay rate small. Thus, what we get with these limits is a lower bound on the vacuum decay rate and it always gives a conservative constraint.

The condition for the stability of the EW vacuum is then given by

$$
\gamma \lesssim H_{0}^{4}
$$

where $H_{0} \simeq 67.66(\mathrm{~km} / \mathrm{s}) / \mathrm{Mpc}[44]$ is the current Hubble constant.

\subsection{Example}

Let us show an example of the calculation. We take

$$
\begin{aligned}
\tan \beta & =9, & \cos (\beta-\alpha) & =0.0004, \\
m_{H} & =602.5 \mathrm{GeV}, & m_{A} & =602.5 \mathrm{GeV}, \quad m_{H^{+}}=600 \mathrm{GeV} .
\end{aligned}
$$

We first calculate the $\overline{\mathrm{MS}}$ dimensionless couplings at renormalization scale $\mu_{t}=m_{t}$, where we include the one-loop corrections and the four-loop QCD corrections. The details are in appendix D. Then, we evolve them with the two-loop RG equations. The result is shown in the top left panel of figure 1. In this example, only $\lambda_{2}$ becomes negative and contributes to the vacuum decay rate.

Next, we calculate the differential vacuum decay rate, $d \gamma / d(\ln R)$, for case (b). We take $\mu=1 / R$. The result is shown with the solid line in the top right panel of figure 1 . Integrating it over $\ln R$, we get

$$
\log _{10}[\gamma \times \text { Gyr Gpc } 3]=-3.5_{-26.1}^{+21.7}{ }_{-11.8}^{+11.0}+1.4{ }_{-0.3}^{+0.1},
$$

where the 1st, 2nd, 3rd and 4th errors are those from $m_{t}, \alpha_{s}, m_{h}$ and $\mu$, respectively. We use the SM values and uncertainties given in table 3 and $\alpha_{s}=0.1181(11)$. We estimate the renormalization scale uncertainty by taking $\mu=2 / R$ and $\mu=1 /(2 R)$. With this parameter set, the vacuum decay rate is close to the upper bound, $\log _{10}\left[H_{0}^{4} \times \mathrm{Gyr} \mathrm{Gpc}^{3}\right] \simeq-3$.

Let us see the difference between the "tree level" vacuum decay rate and our result. For the tree level vacuum decay rate, we adopt

$$
\gamma_{\text {tree }}=\max _{R, \lambda_{\phi}}\left[\frac{1}{R^{4}} e^{-\frac{8 \pi^{2}}{3\left|\lambda_{\phi}\right|}}\right]_{\mu=R^{-1}},
$$



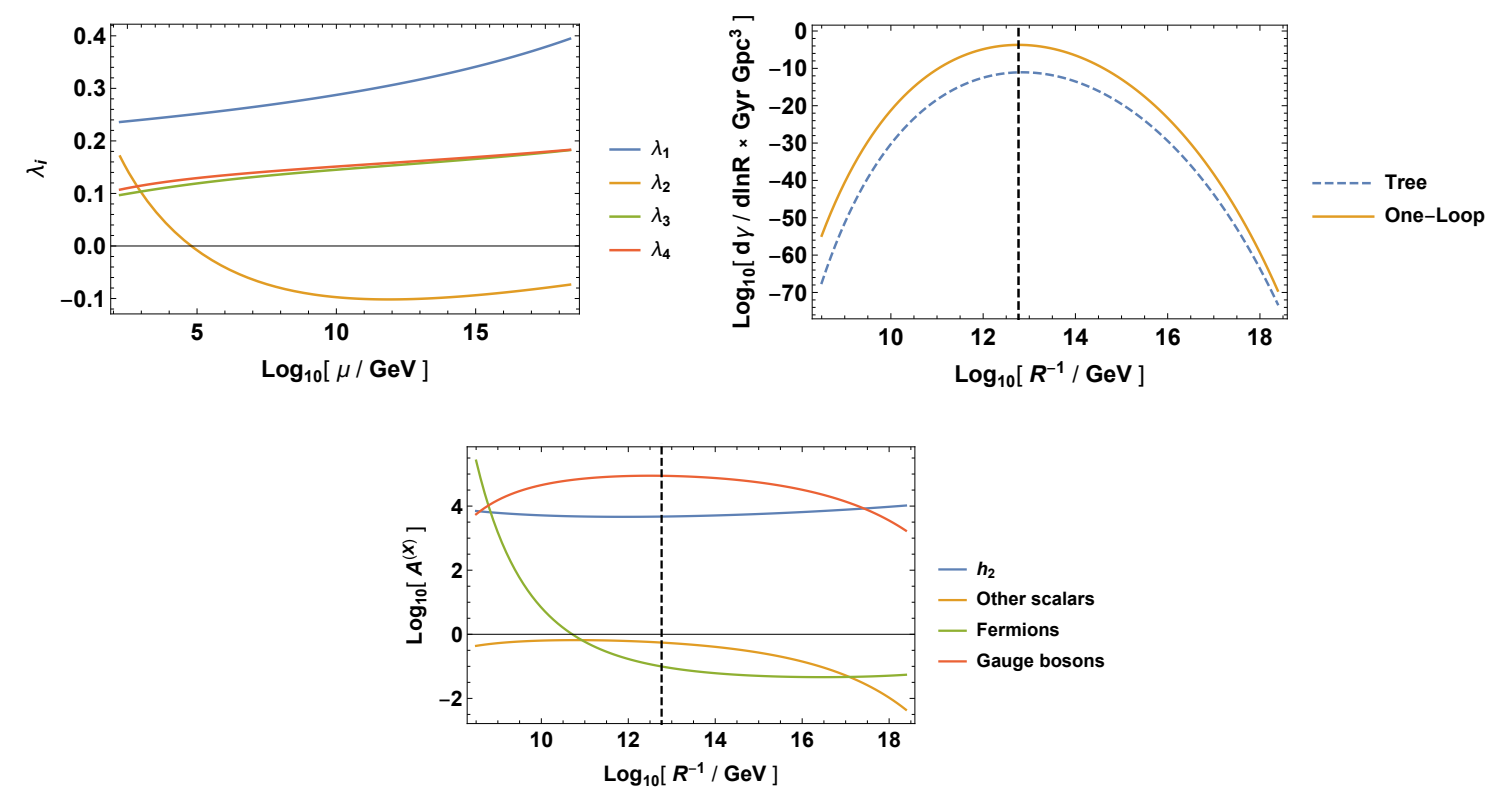

Figure 1. An example of the calculation of a vacuum decay rate. The top left panel shows the RG evolution of the Higgs quartic couplings. The top right panel shows the differential vacuum decay rates with and without the calculation of $\mathcal{A}$. The bottom panel shows each quantum correction to the differential vacuum decay rate. The vertical black dashed line indicates the maximum of the differential vacuum decay rate.

where the maximum value is searched in the same region as the integration region of the oneloop vacuum decay rate. In the top right panel of figure 1 , we show $R^{-4} \exp \left[-8 \pi^{2} /\left(3\left|\lambda_{\phi}\right|\right)\right]$ with the dashed line. We get

$$
\log _{10}\left[\gamma_{\text {tree }} \times \text { Gyr Gpc }{ }^{3}\right]=-11.2 .
$$

Thus, the one-loop calculation enhances the vacuum decay rate by about $10^{7.7}$. We show each quantum contribution in the bottom panel of figure 1 . The vertical black dashed line corresponds to the maximum of the differential vacuum decay rate. Around the maximum, the gauge bosons and $h_{2}$ have positive contributions and the fermions and the other scalars have negative contributions. The former contributions are larger than the latter and the positive contribution remains.

In figure 2, we show the binned plot of the vacuum decay rates at the tree level and at the one-loop level by using the data accumulated for figure 4 . We observe that the enhancement of the vacuum decay rate is generic for $\gamma \gtrsim H_{0}^{4}$ and that it is enhanced at most by $10^{10}$, which is comparable with the uncertainties from those of the top mass and the strong coupling constant. For $\gamma \ll H_{0}^{4}$, the vacuum decay rate can be either suppressed or enhanced.

\section{Low energy constraints}

Before the discussion of the high scale validity, let us discuss the low energy constraints; flavor observables, perturbative unitarity, oblique parameters, and collider searches. In this 


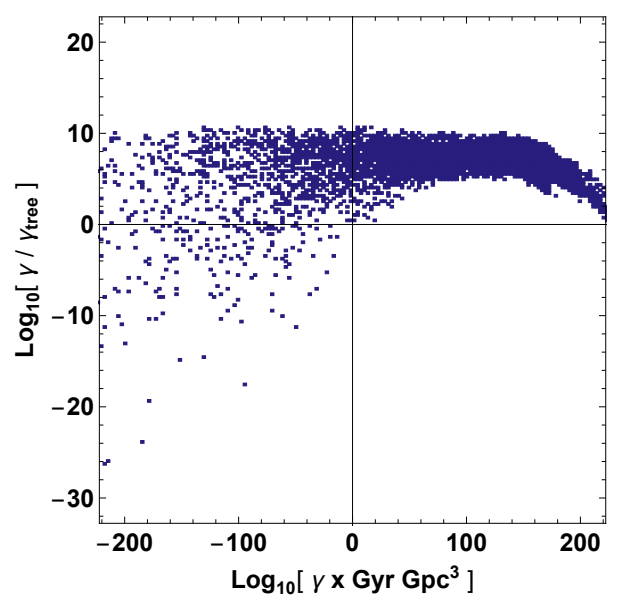

Figure 2. The difference between the tree level vacuum decay rates and the one-loop level vacuum decay rates. We use the data accumulated for figure 4 . We show only $-200 \lesssim \log _{10}[\gamma$ $\left.\times \mathrm{GyrGpc}^{3}\right] \lesssim 200$.

section, we do not consider the constraints from the signal strengths of the $125 \mathrm{GeV}$ Higgs boson since they are the outputs of our analysis.

\subsection{Flavor observables}

The additional Higgs bosons contribute to flavor observables and the current strongest constraints for the type-II THDM come from the branching ratios of $B \rightarrow \tau \nu, B_{s} \rightarrow \mu \mu$ and $b \rightarrow s \gamma$, and the $B_{s}-\bar{B}_{s}$ mixing as discussed, for example, in [45-47]. We obtain the constraints following the analysis of [48] with the current experimental values. The details are given in appendix A.

In figure 3 , we plot the $95 \%$ exclusion limits on the $\left(m_{H^{+}}, \tan \beta\right)$-plane with assuming $m_{H}=m_{A}=m_{H^{+}}$and $\cos (\beta-\alpha)=0 .{ }^{8}$ The white region is allowed and the shaded regions are excluded by the observables shown on the regions. As we can see, $\mathrm{BR}(b \rightarrow s \gamma)$ gives the lower bound of $m_{H^{+}} \gtrsim 580 \mathrm{GeV}$ almost independently of $\tan \beta$. The upper bound and the lower bound on $\tan \beta$ are set by $\operatorname{BR}\left(B_{s} \rightarrow \mu \mu\right)$ and $\Delta M_{B_{s}}$, respectively. Notice that these constraints are stronger than the perturbativity limits of $y_{t}, y_{b} \lesssim \sqrt{4 \pi}$. The results are consistent with the recent works ${ }^{9}[45-47]$.

\subsection{Low energy perturbative unitarity}

For the study of the high scale validity, the perturbative unitarity is necessary because otherwise all the calculations, including the matching conditions to the $\overline{\mathrm{MS}}$ couplings, become unreliable.

\footnotetext{
${ }^{8}$ These parameters affect only $\mathrm{BR}\left(B_{s}^{0} \rightarrow \mu^{+} \mu^{-}\right)$. As we will see later, the high scale validity requires a small $\cos (\beta-\alpha)$ and mass differences. Then, the result is not so much affected as discussed in [48].

${ }^{9}$ Since there are choices of input parameters and of the treatment of theoretical uncertainty, $\mathcal{O}(10 \%)$ difference of the constraints is acceptable.
} 


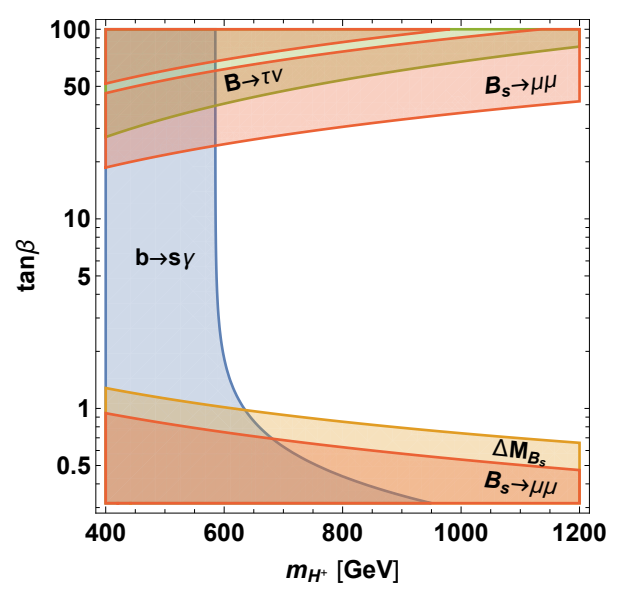

Figure 3. The 95\% CL constraints from flavor observables. The blue, the orange, the red, and the green shaded regions are excluded by $\mathrm{BR}(\mathrm{b} \rightarrow \mathrm{s} \gamma), \Delta M_{B_{s}}, \mathrm{BR}\left(B_{s} \rightarrow \mu \mu\right)$, and $\mathrm{BR}(B \rightarrow \tau \nu)$, respectively.

At the tree level, the Higgs quartic couplings are related to the Higgs masses and mixing as

$$
\begin{aligned}
& \lambda_{1}=\frac{1}{2 v^{2} \cos ^{2} \beta}\left[m_{h}^{2}+m_{H}^{2}-(1-\cos 2 \beta) m_{A}^{2}+\left(m_{H}^{2}-m_{h}^{2}\right) \cos 2 \alpha\right], \\
& \lambda_{2}=\frac{1}{2 v^{2} \sin ^{2} \beta}\left[m_{h}^{2}+m_{H}^{2}-(1+\cos 2 \beta) m_{A}^{2}-\left(m_{H}^{2}-m_{h}^{2}\right) \cos 2 \alpha\right], \\
& \lambda_{3}=\frac{1}{v^{2}}\left[2 m_{H^{+}}^{2}-m_{A}^{2}+\left(m_{H}^{2}-m_{h}^{2}\right) \frac{\sin 2 \alpha}{\sin 2 \beta}\right], \\
& \lambda_{4}=\frac{2}{v^{2}}\left(m_{A}^{2}-m_{H^{+}}^{2}\right) .
\end{aligned}
$$

Then, we impose the condition of the $s$-wave unitarity, which is given by $[49,50]$

$$
\begin{aligned}
\left|\lambda_{1}\right| & <8 \pi, \\
\left|\lambda_{2}\right| & <8 \pi, \\
\left|\lambda_{3}\right| & <8 \pi, \\
\left|\lambda_{3} \pm \lambda_{4}\right| & <8 \pi, \\
\left|\lambda_{3}+2 \lambda_{4}\right| & <8 \pi, \\
\left|\frac{1}{2}\left(\lambda_{1}+\lambda_{2} \pm \sqrt{\left(\lambda_{1}-\lambda_{2}\right)^{2}+4 \lambda_{4}^{2}}\right)\right| & <8 \pi, \\
\left|\frac{3}{2}\left(\lambda_{1}+\lambda_{2} \pm \sqrt{\left(\lambda_{1}-\lambda_{2}\right)^{2}+\frac{4}{9}\left(2 \lambda_{3}+\lambda_{4}\right)^{2}}\right)\right| & <8 \pi,
\end{aligned}
$$

at the tree level. ${ }^{10}$ Since we will use the same condition to detect Landau poles later, we refer to the perturbative unitarity with the tree level matching conditions as "the low energy (LE) perturbative unitarity".

\footnotetext{
${ }^{10}$ Precisely speaking, this condition of perturbative unitarity is valid up to $\mathcal{O}(1)$ uncertainty. However, it is enough for our purpose of avoiding too large quartic couplings.
} 


\subsection{Oblique parameters}

The oblique parameters, especially the $S$-parameter and the $T$-parameter, are affected by the additional Higgs doublet. We use the general formulas for multi-Higgs-doublet models $[51,52]$ (for the THDM, see [53] ${ }^{11}$ ) to calculate these parameters.

The current constraints are given by [54]

$$
\begin{aligned}
& S=0.02 \pm 0.07, \\
& T=0.06 \pm 0.06,
\end{aligned}
$$

with the assumption of $U=0$. The correlation coefficient is $\rho=0.92$. We adopt $95 \%$ exclusion limit on $S$ and $T$, which is given by

$$
\chi_{2 \text { dof }}^{2} \equiv \frac{1}{1-\rho^{2}}\left[\frac{\left(S-S_{\text {cent }}\right)^{2}}{\sigma_{S}^{2}}+\frac{\left(T-T_{\text {cent }}\right)^{2}}{\sigma_{T}^{2}}-2 \rho \frac{\left(S-S_{\text {cent }}\right)\left(T-T_{\text {cent }}\right)}{\sigma_{S} \sigma_{T}}\right]<5.99,
$$

where $S_{\text {cent }}$ and $T_{\text {cent }}$ are the central values of $S$ and $T$, respectively.

\subsection{Collider searches}

New scalar particles have been searched extensively at Tevatron, LEP and LHC. We utilize HiggsBounds [55-59] to check the constraints from the collider searches. For simplicity, we consider only on-shell decays for non-SM channels. The couplings and the partial decay widths used in the analysis are summarized in appendix F.

\section{High scale validity}

At an energy scale much higher than the EW scale, the model becomes classically scale invariant and only the dimensionless couplings become relevant. We first match the $\overline{\mathrm{MS}}$ couplings at the one-loop level, where the matching scale is taken to the top mass scale. For the top and the bottom Yukawa couplings, we also include the four-loop QCD corrections. Then, we evolve the dimensionless couplings up to the Plank scale using the two-loop beta functions. In these calculations, we utilize the public codes of SARAH [60, 61], FeynArts [62], FeynCalc [63, 64], and RunDec [65, 66]. The details of the matching conditions are given in appendix D. Throughout this analysis, we adopt the central values for the SM inputs, which are summarized in table 3.

For the model to be valid up to the Planck scale, Landau poles should not appear during the RG evolution. We adopt the condition of the tree level perturbative unitarity given in eqs. (4.5)-(4.11) to detect Landau poles and require that they should be satisfied until the Planck scale. We refer to this condition as "the high energy (HE) perturbative unitarity". If it is satisfied, we then check the vacuum stability, where we take $\mu=1 / R$.

We reduce the number of free parameters by choosing three slices of parameter space;

$$
\begin{aligned}
\text { (i) } m_{H^{+}}=600 \mathrm{GeV}, & 1.8<\tan \beta<25, \\
\text { (ii) } m_{H^{+}}=900 \mathrm{GeV}, & 0.8<\tan \beta<33, \\
\text { (iii) } m_{H^{+}}=1200 \mathrm{GeV}, & 0.65<\tan \beta<40,
\end{aligned}
$$

\footnotetext{
${ }^{11}$ There is a typo in the $G(x, y, Q)$ function in [53]. The correct definition is in [52].
} 
which satisfy the flavor constraints of figure 3. Since the flavor constraints do not depend so much on the other Higgs masses or $\cos (\beta-\alpha)$ in the region of interest, we do not further check the flavor constraints to reduce computational complexity. In addition, we assume $\sin (\beta-\alpha)>0$ in this analysis.

For each slice, we generate random two million data points that satisfy all of the other low energy constraints, namely, LE perturbative unitarity, oblique parameters and collider searches. The scattering range covers all of the parameter space where the LE perturbative unitarity is satisfied. The details of data generation are in appendix E.

In figure 4 , we show the binned plots of the allowed data points. All the colored points satisfy the low energy constraints. In the upper panels, the large $\tan \beta$ region is excluded by the $H \rightarrow \tau \tau$ channel. For slice (i), the upper and the lower bounds on $\cos (\beta-\alpha)$ are determined by the constraints on the $H \rightarrow V V$ and the $H \rightarrow 2 h \rightarrow 4 b$ channels, respectively. For slices (ii) and (iii), the upper and the lower bounds on $\cos (\beta-\alpha)$ are mostly determined by the constraints on the LE perturbative unitarity and the oblique parameters, respectively. As for the lower panels, the concave shape is due to the constraint on the oblique parameters and the horns have the ends due to the other constraints.

Next, the orange and the green points satisfy the HE perturbative unitarity. The allowed parameter space is reduced especially for slice (i), but the reduction is not so drastic.

Finally, the green points satisfy the vacuum stability condition. As we can see, the parameter space is reduced drastically. It is because of the complementarity of the HE perturbative unitarity and the vacuum stability. It can be understood from the one-loop beta functions of $\lambda_{1}$ and $\lambda_{2}$, which are given by

$$
\begin{aligned}
\beta_{\lambda_{1}}= & 2\left[6 \lambda_{1}^{2}+\lambda_{3}^{2}+\left(\lambda_{3}+\lambda_{4}\right)^{2}\right]+\frac{3}{4}\left(g_{Y}^{4}+3 g_{2}^{4}+2 g_{Y}^{2} g_{2}^{2}\right) \\
& +\lambda_{1}\left(12 y_{b}^{2}+4 y_{\tau}^{2}-3 g_{Y}^{2}-9 g_{2}^{2}\right)-12 y_{b}^{4}-4 y_{\tau}^{4}, \\
\beta_{\lambda_{2}}=2 & {\left[6 \lambda_{2}^{2}+\lambda_{3}^{2}+\left(\lambda_{3}+\lambda_{4}\right)^{2}\right]+\frac{3}{4}\left(g_{Y}^{4}+3 g_{2}^{4}+2 g_{Y}^{2} g_{2}^{2}\right) } \\
& +\lambda_{2}\left(12 y_{t}^{2}-3 g_{Y}^{2}-9 g_{2}^{2}\right)-12 y_{t}^{4} .
\end{aligned}
$$

Since $y_{t}, y_{b}, y_{\tau}$ and $g_{2}$ are UV free, $\beta_{\lambda_{1}}$ and $\beta_{\lambda_{2}}$ generically become positive at a high energy scale. To avoid Landau poles, the quartic couplings should be small enough. In addition, negative $\lambda_{1}$ or $\lambda_{2}$ are preferable since they delay the appearance of Landau poles. Thus, the potential easily becomes unstable and a large part of the parameter space is constrained by the vacuum stability.

A similar condition as the vacuum stability is the bounded-from-below condition, which is given by

$$
\lambda_{1}>0 \& \lambda_{2}>0 \&\left(\frac{\lambda_{1} \lambda_{2}-\bar{\lambda}^{2}}{\lambda_{1}+\lambda_{2}-2 \bar{\lambda}}>0 \text { or } \frac{\lambda_{1}-\bar{\lambda}}{\lambda_{2}-\bar{\lambda}}<0\right)
$$

Here, we regard those couplings as the $\overline{\mathrm{MS}}$ couplings at $\mu=m_{t}$ and impose it only at low energy. Notice that the condition is obtained from the discussion of section 3 and is equivalent to that in [67]. We expect that the combination of the HE perturbative 

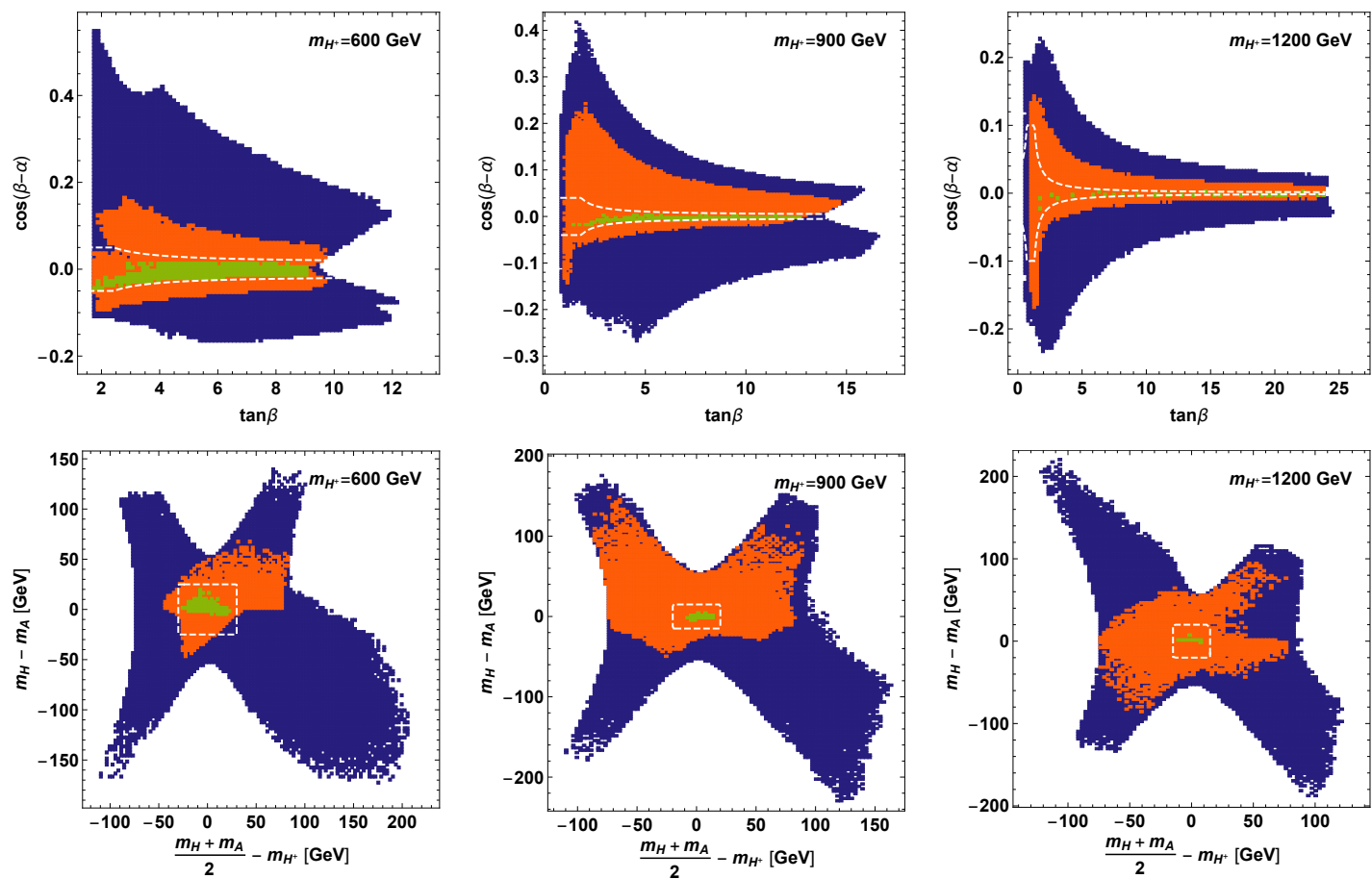

Figure 4. Binned plots of allowed data points. The left, the middle and the right panels correspond to the parameter slices of (i), (ii) and (iii), respectively. All the colored points satisfy the low energy constraints. The orange and the green points satisfy the perturbative unitarity conditions until the Planck scale. The green points satisfy the vacuum stability condition. The regions surrounded by the white dashed lines are used in figure 5 .

unitarity and the bounded-from-below condition should give a similar result, ${ }^{12}$ which we will see below.

In figure 5, we pick up the parameter space defined by the region surrounded by the white dashed lines in figure 4 and prepare additional five million points satisfying all the low energy constraints for each region. The scattering region is taken so that it can cover all the green points. The distribution of the new data points is uniform in the space of $\tan \beta$, $\cos (\beta-\alpha) /|\cos (\beta-\alpha)|_{\max }, m_{H}-m_{A}$ and $\left(m_{H}+m_{A}\right) / 2-m_{H^{+}}$. Here, $|\cos (\beta-\alpha)|_{\max }$ is the maximum value of $\cos (\beta-\alpha)$ depending on $\tan \beta$, which is shown in figure 4 . The red points satisfy the bounded-from-below condition and the HE perturbative unitarity. The lighter and the darker green points correspond to the green points in figure 4 and are plotted over the red points. Thus, in the red region appearing in the figure, the potential is stable at low energy, but always becomes unstable at high energy. The darker green points satisfy both the vacuum stability and the bounded-from-below conditions. Thus, in the lighter green region, the potential always becomes unstable at low energy, but the instability is cured at high energy. Notice that the vacuum decay rates can be affected by the IR cut-off for the $R$ integral in the lighter green region.

\footnotetext{
${ }^{12}$ If we impose only the bounded-from-below condition and the low energy constraints, the allowed region is as large as the orange region of figure 4 .
} 

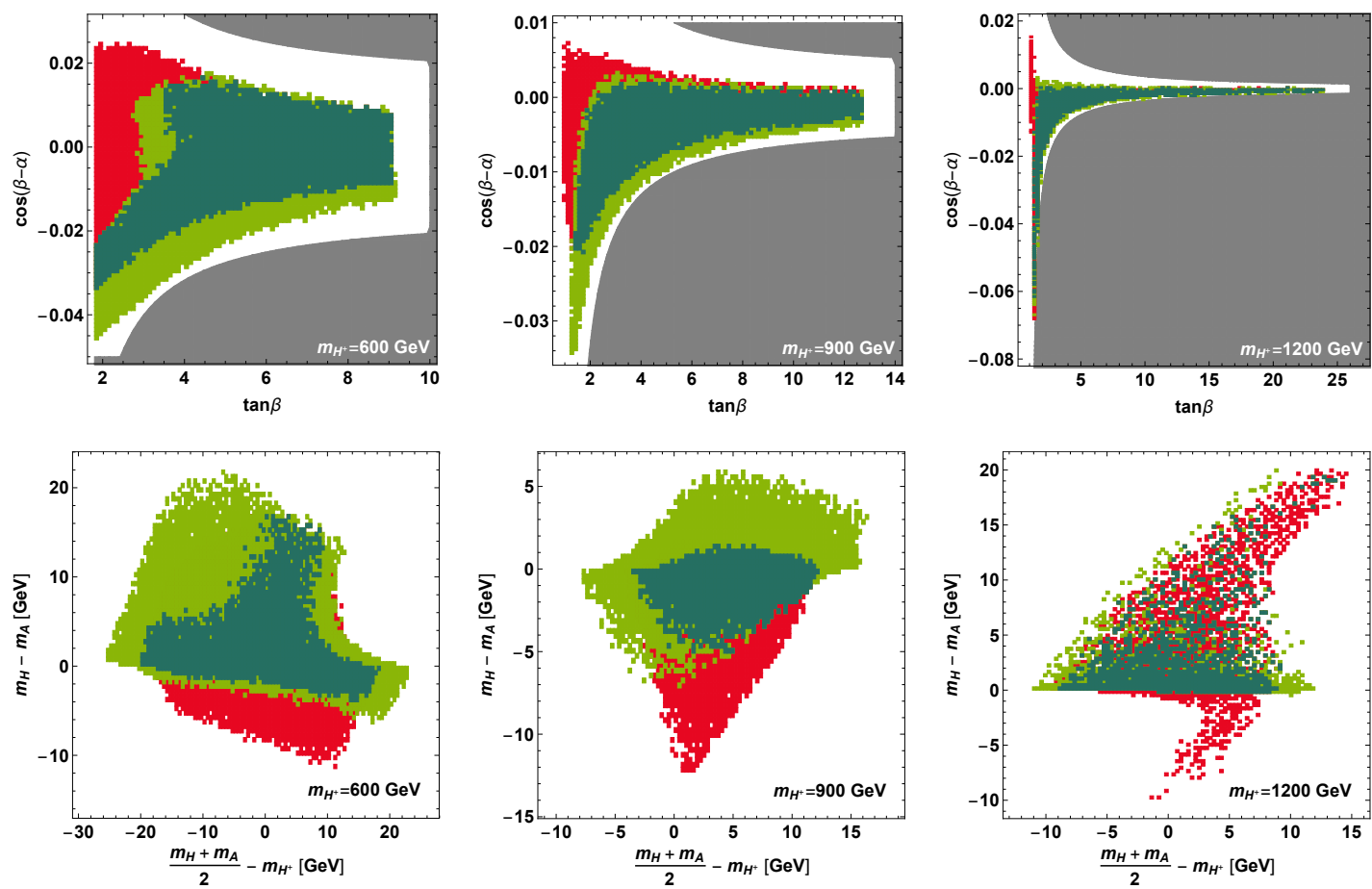

Figure 5. The detail views of the parameter regions inside the white dashed lines in figure 4 . The masked region is shaded with gray. All the points satisfy the low energy constraints and remain perturbative until the Planck scale. The red points satisfy the bounded-from-below constraint. The lighter green points satisfy the vacuum stability constraint. The darker green points satisfy both of them.

As we can see from the figure, the bounded-from-below condition has a similar effect as the vacuum stability condition, but the allowed regions do not overlap completely. In particular, a large part of the region with $m_{H}<m_{A}$ is excluded by the vacuum stability, where $\lambda_{2}$ tends to become negative during the RG evolution. In addition, a negative $\cos (\beta-\alpha)$ is more favored by the vacuum stability.

Let us discuss the implication on the Higgs couplings. At the tree level, the SM-value normalized couplings of the $125 \mathrm{GeV}$ Higgs boson are given by

$$
\begin{aligned}
g_{h U U} & =\sin (\beta-\alpha)+\cot \beta \cos (\beta-\alpha), \\
g_{h D D} & =\sin (\beta-\alpha)-\tan \beta \cos (\beta-\alpha), \\
g_{h L L} & =\sin (\beta-\alpha)-\tan \beta \cos (\beta-\alpha), \\
g_{h V V} & =\sin (\beta-\alpha),
\end{aligned}
$$

where $U, D, L$, and $V$ represent the up-type quarks, the down-type quarks, the leptons, and the gauge bosons, respectively. Since $|\cos (\beta-\alpha)| \lesssim 0.06$ for all the slices, we have $0.9982 \lesssim g_{h V V} \leq 1$, which is not possible to be distinguished from unity even with HL-LHC plus $1 \mathrm{TeV}$ ILC [68]. It also means that the model cannot be valid up to the Planck scale if we observe larger deviations of $g_{h V V}$ couplings. On the other hand, the other couplings can deviate by more than $1 \%$ because of the second term of the above equations. 

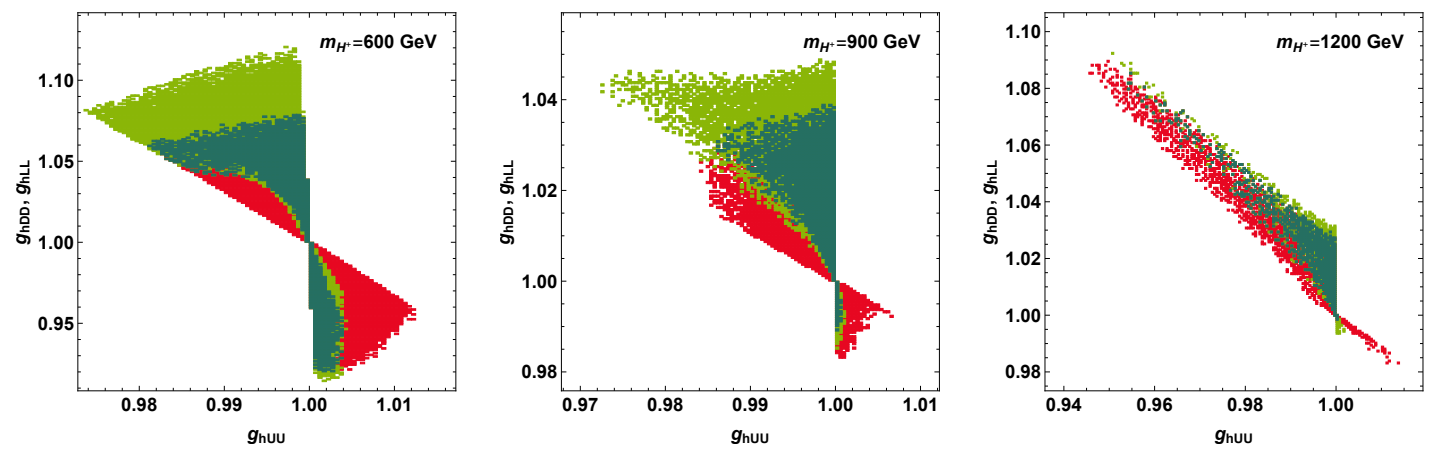

Figure 6. The SM-normalized couplings of the $125 \mathrm{GeV}$ Higgs boson. The colors are the same as in figure 5 .

In figure 6, we cast the data points in figure 5 into the $g_{h U U}$ vs $g_{h D D}=g_{h L L}$ plane. The colors are the same as in figure 5 . As we can see, $g_{h U U}$ can be reduced by about $2 \%-5 \%$ for each slice, but cannot be enhanced so much. On the other hand, $g_{h D D}$ and $g_{h L L}$ can deviate by about $5 \%-12 \%$ for each slice, and tend to be enhanced.

The current constraints on these couplings are given by [69]

$$
\begin{aligned}
g_{h Z Z} & =1.10 \pm 0.08, \\
g_{h W W} & =1.05 \pm 0.08, \\
g_{h b b} & =1.06_{-0.18}^{+0.19}, \\
g_{h t t} & =1.02_{-0.10}^{+0.11}, \\
g_{h \tau \tau} & =1.07 \pm 0.15,
\end{aligned}
$$

with the assumption that there is no new particles in loops and decays. Thus, they have already started to touch the parameter space. Future measurements of the Higgs couplings by, for example, the combination of HL-LHC and ILC will reach the precision of a few percent level [68] and will possibly find deviations from the SM values.

Let us discuss the dependence on the IR cut-off and the UV cut-off, which are introduced in eqs. (3.21) and (3.22). Since the beta functions for $\lambda_{1}$ and $\lambda_{2}$ become generically positive at high energy, a factor change of the UV cut-off rarely affects the vacuum decay rate, which we have checked numerically as well. As for the IR cut-off, we have checked that figures 5 and 6 are not affected even if we use $1 \mathrm{TeV}$ for the IR cut-off, instead of $10 \mathrm{TeV}$.

Finally, we comment on the effect of $\mathcal{A}$, which we have calculated precisely. Although the vacuum decay rates are enhanced compared with the tree level ones around $\gamma \sim H_{0}^{4}$, we find that the effect is not large enough to change figure 6 . It is because of the strong dependence of the vacuum decay rates on the Higgs quartic couplings. However, if we find the additional Higgs bosons in future, the vacuum decay rate can be determined precisely from the measurements of the mass differences and the couplings of the Higgs bosons, which will give an important implication on the scenario. 


\section{Summary}

In this paper, we analyzed the high scale validity of the DFSZ axion model, namely the HE perturbative unitarity and the vacuum stability. The model has been widely studied since it can explain the strong CP problem and dark matter elegantly. Once we admit a mechanism that forces classical scale invariance at the Planck scale, the Higgs mass terms of the appropriate size can be generated through the technically natural parameters and may be valid up to the Planck scale. In addition, the model can be extended without affecting the Higgs sector to explain the neutrino masses, the baryon asymmetry of the Universe and inflation. Thus, the high scale behavior of the Higgs sector is worth discussing.

We utilized the state-of-the-art method to calculate the vacuum decay rate precisely. We extended the results of [39-41] to accommodate bounces that are composed of more than one fields. Then, we showed that $\mathcal{A}$ can enhance the vacuum decay rates at most by $10^{10}$, which can become comparable with the uncertainties from those of the top mass and the strong coupling constant.

We performed the parameter scan and found the parameter space that satisfies the constraints from flavor observables, LE/HE perturbative unitarity, oblique parameters, collider searches, and vacuum stability. Due to the complementarity of the HE perturbative unitarity and the vacuum stability, the allowed parameter space becomes very small. We observe that it still accommodates at most $12 \%$ enhancement of the $h D D$ and $h L L$ couplings, and at most $5 \%$ suppression of the $h U U$ couplings. These are around the current experimental constraints and will be searched at future experiments such as HL-LHC and ILC. On the other hand, the deviation of the $h V V$ couplings are found to be smaller than $0.2 \%$ and the scenario may be excluded if we observe large deviations of these couplings.

\section{Acknowledgments}

Y.S. is supported by Grant-in-Aid for Scientific research from the Ministry of Education, Science, Sports, and Culture (MEXT), Japan, No. 16H06492. S.O. and D.-s.T. are supported by the mathematical and theoretical physics unit (Hikami unit) of the Okinawa Institute of Science and Technology Graduate University. S.O. and D.-s.T. are also supported by Japan Society for the Promotion of Science (JSPS), Grant-in-Aid for Scientific Research (C), Grant Number JP18K03661. The authors thank the Yukawa Institute for Theoretical Physics at Kyoto University, where this work was initiated during the YITPW-18-05 on "Progress in Particle Physics 2018 (PPP2018)".

\section{A Flavor}

In this appendix, we follow [48] and obtain the flavor constraints with the current experimental values.

\section{A.1 CKM matrix elements}

We first determine the CKM matrix elements by using observables that are insensitive to the additional Higgs bosons. 


\begin{tabular}{|c|c|}
\hline \multicolumn{2}{|r|}{ Mesons } \\
\hline Input & Value \\
\hline$m_{K^{ \pm}}$ & $493.677 \mathrm{MeV}[54]$ \\
\hline$m_{B^{ \pm}}$ & $5.27933 \mathrm{GeV}[54]$ \\
\hline$m_{B_{s}}$ & $5.36688 \mathrm{GeV}[54]$ \\
\hline$\tau_{K^{ \pm}}$ & $12.38 \pm 0.02 \mathrm{~ns}[54]$ \\
\hline$\tau_{B^{ \pm}}$ & $1.638 \pm 0.004 \mathrm{ps}[54]$ \\
\hline$\tau_{B_{s}}^{H}$ & $1.619 \pm 0.009 \mathrm{ps}[54]$ \\
\hline$\tau_{B_{s}}^{L}$ & $1.414 \pm 0.006 \mathrm{ps}[54]$ \\
\hline
\end{tabular}

\begin{tabular}{|cc|}
\hline \multicolumn{2}{|c|}{ Theoretical Inputs } \\
\hline Input & Value \\
\hline $\mathrm{BR}(b \rightarrow s \gamma)_{E_{\gamma}>1.6 \mathrm{GeV}}$ & $(3.36 \pm 0.23) \times 10^{-4}[72,73]$ \\
$f_{K}$ & $155.7 \pm 0.3 \mathrm{MeV}[74]$ \\
$f_{B}$ & $190.0 \pm 1.3 \mathrm{MeV}[74]$ \\
$f_{B_{s}}$ & $230.3 \pm 1.3 \mathrm{MeV}[74]$ \\
$f_{B_{s}}^{2} B_{2}^{(s)}\left(m_{b}\right)$ & $0.0421 \pm 0.0028 \mathrm{GeV}^{2}[75]$ \\
$f_{B_{s}}^{2} B_{3}^{(s)}\left(m_{b}\right)$ & $0.0576 \pm 0.0078 \mathrm{GeV}^{2}[75]$ \\
$\hat{B}_{B_{s}}$ & $1.35 \pm 0.06[74]$ \\
\hline
\end{tabular}

Table 2. Experimental and theoretical parameters for mesons. The uncertainties are used for the evaluation of theoretical uncertainties in the flavor analysis.

We use the Wolfenstein parametrization defined as

$$
V_{\mathrm{CKM}}=\left(\begin{array}{ccc}
1-\frac{\lambda_{\mathrm{CKM}}^{2}}{2} & \lambda_{\mathrm{CKM}} & A_{\mathrm{CKM}} \lambda_{\mathrm{CKM}}^{3}\left(\rho_{\mathrm{CKM}}-i \eta_{\mathrm{CKM}}\right) \\
-\lambda_{\mathrm{CKM}} & 1-\frac{\lambda_{\mathrm{CKM}}^{2}}{2} & A_{\mathrm{CKM}} \lambda_{\mathrm{CKM}}^{2} \\
A_{\mathrm{CKM}} \lambda_{\mathrm{CKM}}^{3}\left(1-\rho_{\mathrm{CKM}}-i \eta_{\mathrm{CKM}}\right) & -A_{\mathrm{CKM}} \lambda_{\mathrm{CKM}}^{2} & 1
\end{array}\right),
$$

where we neglect $\mathcal{O}\left(\lambda_{\text {CKM }}^{4}\right)$. We determine $\lambda_{\text {CKM }}$ by using the super allowed nuclear beta decays, $\left|V_{u d}\right|=0.97420 \pm 0.00021[70]$, and the $K \rightarrow e \nu$ decay, $\operatorname{BR}(K \rightarrow e \nu)=(1.582 \pm$ $0.007) \times 10^{-5}[54]$. Combining these two, we obtain

$$
\lambda_{\mathrm{CKM}}=0.2244 \pm 0.0005 .
$$

Here, we have used experimental values for the $K$ meson in table 2 .

Next, we determine $A_{\mathrm{CKM}}$ from $\left|V_{c b}\right|$ assuming that the corrections from the charged Higgs boson are small, which is justified for $m_{H^{+}}>150 \mathrm{GeV}$ and $\tan \beta<100$ [48]. We use $\left|V_{c b}\right|=(39.25 \pm 0.56) \times 10^{-3}[71]$ and get

$$
A_{\mathrm{CKM}}=0.779 \pm 0.012 \text {. }
$$

Finally, we determine $\rho_{\mathrm{CKM}}$ and $\eta_{\mathrm{CKM}}$ from the unitary triangle. From $\phi_{1}=(22.2 \pm$ $0.7)^{\circ}, \phi_{2}=(84.9 \pm 5)^{\circ}$, and $\phi_{3}=(71.1 \pm 5)^{\circ}[71]$, we get

$$
\begin{aligned}
& \bar{\rho}_{\mathrm{CKM}}=0.117 \pm 0.020, \\
& \bar{\eta}_{\mathrm{CKM}}=0.361 \pm 0.012,
\end{aligned}
$$

where

$$
\rho_{\mathrm{CKM}}+i \eta_{\mathrm{CKM}}=\frac{\bar{\rho}_{\mathrm{CKM}}+i \bar{\eta}_{\mathrm{CKM}}}{1-A_{\mathrm{CKM}}^{2} \lambda_{\mathrm{CKM}}^{4}\left(\bar{\rho}_{\mathrm{CKM}}+i \bar{\eta}_{\mathrm{CKM}}\right)} \sqrt{\frac{1-A_{\mathrm{CKM}}^{2} \lambda_{\mathrm{CKM}}^{4}}{1-\lambda_{\mathrm{CKM}}^{2}}} .
$$




\begin{tabular}{|c|c|c|c|}
\hline \multicolumn{2}{|r|}{ EW Parameters } & \multicolumn{2}{|c|}{ Fermion Masses } \\
\hline Input & Value & Input & Value \\
\hline$m_{h}$ & $125.1 \pm 0.14 \mathrm{GeV}[54]$ & $M_{t}$ & $173.1 \pm 0.9 \mathrm{GeV}[54]$ \\
\hline$m_{W}$ & $80.379 \mathrm{GeV}[54]$ & $m_{b}\left(m_{b}\right)$ & $4.198 \pm 0.012 \mathrm{GeV}[74]$ \\
\hline$m_{Z}$ & $91.1876 \mathrm{GeV}[54]$ & $m_{s}(2 \mathrm{GeV})$ & $93.44 \pm 0.68 \mathrm{MeV}[74]$ \\
\hline$\alpha_{s}\left(m_{Z}\right)$ & $0.1181[54]$ & $m_{u}(2 \mathrm{GeV})$ & $2.50 \pm 0.17 \mathrm{MeV}[74]$ \\
\hline$G_{F}$ & $1.1663787 \times 10^{-5} \mathrm{GeV}^{-2}[54]$ & $m_{\mu}$ & $105.6583745 \mathrm{MeV}[54]$ \\
\hline $\sin ^{2} \theta_{W}\left(m_{Z}\right)$ & $0.23122[54]$ & $m_{\tau}$ & $1.77686 \mathrm{GeV}[54]$ \\
\hline
\end{tabular}

Table 3. Fundamental parameters of the SM. The uncertainties are used for the evaluation of theoretical uncertainties in the flavor analysis. The top mass is the on-shell mass and the other quark masses are the $\overline{\mathrm{MS}}$ masses with the renormalization scale shown in the parentheses.

\begin{tabular}{|cc|}
\hline \multicolumn{2}{|c|}{ Experimental Results } \\
\hline Observable & Value \\
\hline $\mathrm{BR}(b \rightarrow s \gamma)_{E_{\gamma}>1.6 \mathrm{GeV}}$ & $(3.32 \pm 0.15) \times 10^{-4}[71]$ \\
$\mathrm{BR}(B \rightarrow \tau \nu)$ & $(1.06 \pm 0.19) \times 10^{-4}[71]$ \\
$\mathrm{BR}\left(B_{s} \rightarrow \mu \mu\right)$ & $(3.1 \pm 0.6) \times 10^{-9}[71]$ \\
$\Delta M_{B_{s}}$ & $17.757 \pm 0.021 \mathrm{ps}^{-1}[71]$ \\
\hline
\end{tabular}

Table 4. Experimental results for flavor observables.

\section{A.2 Flavor constraints}

For the theoretical evaluation of the flavor observables, we use the formulas given in [48]. In the calculation, we utilize RunDec $[65,66]$ to get the running masses of quarks.

Let us clarify the statistical method that we adopt. For observable $X$ that depends on known parameters $\left\{x_{i} \pm \delta x_{i}\right\}$ and model parameters $\left\{y_{i}\right\}$, we define

$$
\chi^{2}\left(\left\{y_{i}\right\}\right)=\frac{\left(X\left(\left\{y_{i}\right\}\right)-X_{\exp }\right)^{2}}{\delta X_{\mathrm{th}}^{2}\left(\left\{y_{i}\right\}\right)+\delta X_{\exp }^{2}},
$$

where $X_{\exp } \pm \delta X_{\exp }$ is the experimental result, $X\left(\left\{y_{i}\right\}\right)$ is the theoretical result for inputs $\left\{x_{i}\right\}$ and $\left\{y_{i}\right\}$, and

$$
\delta X_{\mathrm{th}}^{2}\left(\left\{y_{i}\right\}\right)=\sum_{k}\left[\left.X\left(\left\{y_{i}\right\}\right)\right|_{x_{k} \rightarrow x_{k}+\delta x_{k} / 2}-\left.X\left(\left\{y_{i}\right\}\right)\right|_{x_{k} \rightarrow x_{k}-\delta x_{k} / 2}\right]^{2} .
$$

Then, we use the offset corrected $\chi^{2}$ defined as

$$
\Delta \chi^{2}\left(\left\{y_{i}\right\}\right)=\chi^{2}\left(\left\{y_{i}\right\}\right)-\min _{\left\{\tilde{y}_{i}\right\}} \chi^{2}\left(\left\{\tilde{y}_{i}\right\}\right)
$$


and the $95 \%$ CL exclusion limit is given by $\Delta \chi^{2}\left(\left\{y_{i}\right\}\right) \lesssim 3.84$. Here, the minimum value is searched over the parameter space of figure 3 and the SM limit. The known parameters $\left\{x_{i} \pm \delta x_{i}\right\}$ are summarized in tables 2 and 3 , where we ignore uncertainties of $\mathcal{O}(0.1 \%) .{ }^{13}$

The result is shown in figure 3 .

\section{B Proof of straight bounce}

In this appendix, we show that $\Omega$ and $\Theta$ of the relevant solution do not depend on $r$. In terms of $\phi, \Omega$ and $\Theta$, the Euclidean action is expressed as

$$
\begin{aligned}
S_{E}[\phi, \Omega, \Theta] & =\mathcal{K}[\phi, \Omega, \Theta]+\mathcal{V}[\phi, \Omega, \Theta], \\
\mathcal{K}[\phi, \Omega, \Theta] & =2 \pi^{2} \int d r r^{3}\left[\frac{1}{2} \phi^{\prime 2}+\frac{1}{2} \phi^{2}\left(\Omega^{\prime 2}+\Theta^{\prime 2} \sin ^{2} \Omega\right)\right], \\
\mathcal{V}[\phi, \Omega, \Theta] & =2 \pi^{2} \int d r r^{3} \frac{\lambda_{\phi}}{4} \phi^{4},
\end{aligned}
$$

where $\Omega^{\prime}, \Theta^{\prime}$ and $\phi^{\prime}$ are derivatives with respect to $r$. As introduced in [76], we can obtain the bounce by minimizing $\mathcal{K}[\tilde{\phi}, \tilde{\Omega}, \tilde{\Theta}]$ with the constraint given by

$$
\mathcal{V}[\tilde{\phi}, \tilde{\Omega}, \tilde{\Theta}]=(\text { const. })<0 .
$$

After the minimization, the bounce solution is obtained as

$$
\phi(r)=\tilde{\phi}(\sigma r), \Omega(r)=\tilde{\Omega}(\sigma r), \Theta(r)=\tilde{\Theta}(\sigma r),
$$

where

$$
\sigma=\sqrt{-\frac{2 \mathcal{V}[\tilde{\phi}, \tilde{\Omega}, \tilde{\Theta}]}{\mathcal{K}[\tilde{\phi}, \tilde{\Omega}, \tilde{\Theta}]}}
$$

Its Euclidean action is given by

$$
S_{E}=\frac{\mathcal{K}[\tilde{\phi}, \tilde{\Omega}, \tilde{\Theta}]}{2 \sigma^{2}}
$$

Let us assume that there exists a minimum, $\mathcal{K}\left[\tilde{\phi}_{A}, \tilde{\Omega}_{A}, \tilde{\Theta}_{A}\right]$, where $\tilde{\Omega}_{A}$ or $\tilde{\Theta}_{A}$ is not constant. Since $\lambda_{\phi}(\Omega, \Theta)$ is a continuous function, there exist constant $\Omega_{B}$ and $\Theta_{B}$ satisfying

$$
\mathcal{V}\left[\tilde{\phi}_{A}, \tilde{\Omega}_{A}, \tilde{\Theta}_{A}\right]=\mathcal{V}\left[\tilde{\phi}_{A}, \Omega_{B}, \Theta_{B}\right]
$$

Then, we have

$$
\mathcal{K}\left[\tilde{\phi}_{A}, \tilde{\Omega}_{A}, \tilde{\Theta}_{A}\right]-\mathcal{K}\left[\tilde{\phi}_{A}, \Omega_{B}, \Theta_{B}\right]=2 \pi^{2} \int d r r^{3} \frac{1}{2} \tilde{\phi}_{A}^{2}\left(\tilde{\Omega}_{A}^{\prime 2}+\tilde{\Theta}_{A}^{\prime 2} \sin ^{2} \tilde{\Omega}_{A}\right) \geq 0
$$

The equality holds only when $\tilde{\Omega}^{\prime}(r)=\tilde{\Theta}^{\prime}(r)=0$ for any $r$. Notice that when $\sin \tilde{\Omega}_{A}=0$, the field space is not parameterized by $\tilde{\Theta}_{A}$. Then, from eq. (B.7), there exists a bounce with smaller action if $\tilde{\Omega}$ or $\tilde{\Theta}$ is not constant. Thus, the bounce with minimum action can only be realized with constant $\Omega$ and $\Theta .{ }^{14}$

\footnotetext{
${ }^{13}$ We have also ignored the uncertainty of the strong coupling constant since its effect is suppressed compared with other uncertainties.

${ }^{14}$ Only the bounce with minimum action is relevant for the vacuum decay since the contributions from the others are exponentially suppressed.
} 


\section{One-loop corrections to a vacuum decay rate}

From [41], the differential vacuum decay rate is expressed as

$$
\frac{d \gamma}{d R}=\left.\frac{1}{R^{5}} \mathcal{A}^{(h)} \mathcal{A}^{(\sigma)} \mathcal{A}^{(\psi)} \mathcal{A}^{\left(A_{\mu}, \varphi\right)} e^{-\mathcal{B}}\right|_{\mu \sim R^{-1}},
$$

where

$$
\begin{aligned}
\ln \mathcal{A}^{\prime(h)} & =\left.\left[\ln \mathcal{A}^{\prime(h)}\right]_{\overline{\mathrm{MS}}}\right|_{\lambda \rightarrow \lambda_{\phi}}, \\
\ln \mathcal{A}^{(\sigma)} & =\left.\sum_{i} n_{i}^{(\sigma)}\left[\ln \mathcal{A}^{(\sigma)}\right]_{\overline{\mathrm{MS}}}\right|_{\kappa \rightarrow \kappa_{i}, \lambda \rightarrow \lambda_{\phi}}, \\
\ln \mathcal{A}^{(\psi)} & =\left.\sum_{i} n_{i}^{(\psi)}\left[\ln \mathcal{A}^{(\psi)}\right]_{\overline{\mathrm{MS}}}\right|_{y \rightarrow y_{i}, \lambda \rightarrow \lambda_{\phi}}, \\
\ln \mathcal{A}^{\left(A_{\mu}, \varphi\right)} & =\ln \mathcal{V}_{G}+\left.\sum_{i} n_{i}^{\left(A_{\mu}, \varphi\right)}\left[\ln \mathcal{A}^{\prime\left(A_{\mu}, \varphi\right)}\right]_{\overline{\mathrm{MS}}}\right|_{g^{2} \rightarrow \tilde{g}_{i}^{2}, \lambda \rightarrow \lambda_{\phi}} .
\end{aligned}
$$

Here, $\left[\ln \mathcal{A}^{(X)}\right]_{\overline{\mathrm{MS}}}$ 's are defined in [41]. The degrees of freedom, $n_{i}^{(X)}$, and the couplings, $\kappa_{i}, y_{i}$ and $g_{i}^{2}$, are summarized below for each case. For case (c), the symmetry breaking pattern depends on the sign of $\lambda_{4}$. Thus, we divide it into two cases; (c.1): $\lambda_{4}<0$ and (c.2): $\lambda_{4}>0$.

Case (a) $\lambda_{\phi}=\frac{1}{2} \lambda_{1}$

The scalar contributions:

$$
\begin{array}{ll}
n_{1}^{(\sigma)}=2, & \kappa_{1}=\frac{\lambda_{3}}{2}, \\
n_{2}^{(\sigma)}=2, & \kappa_{2}=\frac{\lambda_{3}+\lambda_{4}}{2} .
\end{array}
$$

The fermion contributions:

$$
\begin{array}{ll}
n_{1}^{(\psi)}=3, & y_{1}=y_{b}, \\
n_{2}^{(\psi)}=1, & y_{2}=y_{\tau} .
\end{array}
$$

The gauge boson contributions:

$$
\begin{array}{ll}
n_{1}^{\left(A_{\mu}, \varphi\right)}=2, & \tilde{g}_{1}^{2}=\frac{g_{2}^{2}}{4}, \\
n_{2}^{\left(A_{\mu}, \varphi\right)}=1, & \tilde{g}_{2}^{2}=\frac{g_{Y}^{2}+g_{2}^{2}}{4} .
\end{array}
$$

Case (b) $\lambda_{\phi}=\frac{1}{2} \lambda_{2}$

The scalar contributions:

$$
\begin{array}{ll}
n_{1}^{(\sigma)}=2, & \kappa_{1}=\frac{\lambda_{3}}{2}, \\
n_{2}^{(\sigma)}=2, & \kappa_{2}=\frac{\lambda_{3}+\lambda_{4}}{2} .
\end{array}
$$


The fermion contributions:

$$
n_{1}^{(\psi)}=3, \quad y_{1}=y_{t} .
$$

The gauge boson contributions:

$$
\begin{array}{ll}
n_{1}^{\left(A_{\mu}, \varphi\right)}=2, & \tilde{g}_{1}^{2}=\frac{g_{2}^{2}}{4}, \\
n_{2}^{\left(A_{\mu}, \varphi\right)}=1, & \tilde{g}_{2}^{2}=\frac{g_{Y}^{2}+g_{2}^{2}}{4} .
\end{array}
$$

Case (c.1) $\lambda_{\phi}=\frac{1}{2} \frac{\lambda_{1} \lambda_{2}-\left(\lambda_{3}+\lambda_{4}\right)^{2}}{\lambda_{1}+\lambda_{2}-2\left(\lambda_{3}+\lambda_{4}\right)}$

The scalar contributions:

$$
\begin{array}{ll}
n_{1}^{(\sigma)}=2, & \kappa_{1}=\lambda_{\phi}+\frac{\left|\lambda_{4}\right|}{2}, \\
n_{2}^{(\sigma)}=1, & \kappa_{2}=\lambda_{\phi}+\frac{\left(\lambda_{3}+\lambda_{4}-\lambda_{1}\right)\left(\lambda_{3}+\lambda_{4}-\lambda_{2}\right)}{\lambda_{1}+\lambda_{2}-2\left(\lambda_{3}+\lambda_{4}\right)}, \\
n_{3}^{(\sigma)}=1, & \kappa_{3}=\lambda_{\phi} .
\end{array}
$$

The fermion contributions:

$$
\begin{array}{ll}
n_{1}^{(\psi)}=3, & y_{1}=y_{t} \sqrt{\frac{\lambda_{1}-\lambda_{3}-\lambda_{4}}{\lambda_{1}+\lambda_{2}-2\left(\lambda_{3}+\lambda_{4}\right)}}, \\
n_{2}^{(\psi)}=3, & y_{2}=y_{b} \sqrt{\frac{\lambda_{2}-\lambda_{3}-\lambda_{4}}{\lambda_{1}+\lambda_{2}-2\left(\lambda_{3}+\lambda_{4}\right)}}, \\
n_{3}^{(\psi)}=1, & y_{3}=y_{\tau} \sqrt{\frac{\lambda_{2}-\lambda_{3}-\lambda_{4}}{\lambda_{1}+\lambda_{2}-2\left(\lambda_{3}+\lambda_{4}\right)}} .
\end{array}
$$

The gauge boson contributions:

$$
\begin{array}{ll}
n_{1}^{\left(A_{\mu}, \varphi\right)}=2, & \tilde{g}_{1}^{2}=\frac{g_{2}^{2}}{4}, \\
n_{2}^{\left(A_{\mu}, \varphi\right)}=1, & \tilde{g}_{2}^{2}=\frac{g_{Y}^{2}+g_{2}^{2}}{4} .
\end{array}
$$

Case (c.2) $\lambda_{\phi}=\frac{1}{2} \frac{\lambda_{1} \lambda_{2}-\lambda_{3}^{2}}{\lambda_{1}+\lambda_{2}-2 \lambda_{3}}$

The scalar contributions:

$$
\begin{array}{ll}
n_{1}^{(\sigma)}=2, & \kappa_{1}=\lambda_{\phi}+\frac{\left|\lambda_{4}\right|}{2} \\
n_{2}^{(\sigma)}=1, & \kappa_{2}=\lambda_{\phi}+\frac{\left(\lambda_{3}-\lambda_{1}\right)\left(\lambda_{3}-\lambda_{2}\right)}{\lambda_{1}+\lambda_{2}-2 \lambda_{3}} .
\end{array}
$$

The fermion contributions:

$$
\begin{aligned}
n_{1}^{(\psi)} & =3, & y_{1} & =\sqrt{y_{t}^{2} \frac{\lambda_{1}-\lambda_{3}}{\lambda_{1}+\lambda_{2}-2 \lambda_{3}}+y_{b}^{2} \frac{\lambda_{2}-\lambda_{3}}{\lambda_{1}+\lambda_{2}-2 \lambda_{3}}}, \\
n_{2}^{(\psi)} & =1, & y_{2} & =y_{\tau} \sqrt{\frac{\lambda_{2}-\lambda_{3}}{\lambda_{1}+\lambda_{2}-2 \lambda_{3}}} .
\end{aligned}
$$


The gauge boson contributions:

$$
\begin{array}{ll}
n_{1}^{\left(A_{\mu}, \varphi\right)}=2, & \tilde{g}_{1}^{2}=\frac{g_{2}^{2}}{4}, \\
n_{2}^{\left(A_{\mu}, \varphi\right)}=1, & \tilde{g}_{2}^{2}=\frac{g_{Y}^{2}+g_{2}^{2}+\sqrt{\left(g_{Y}^{2}-g_{2}^{2}\right)^{2}+4 g_{Y}^{2} g_{2}^{2}\left(\frac{\lambda_{1}-\lambda_{2}}{\lambda_{1}+\lambda_{2}-2 \lambda_{3}}\right)^{2}}}{8}, \\
n_{3}^{\left(A_{\mu}, \varphi\right)}=1, & \tilde{g}_{3}^{2}=\frac{g_{Y}^{2}+g_{2}^{2}-\sqrt{\left(g_{Y}^{2}-g_{2}^{2}\right)^{2}+4 g_{Y}^{2} g_{2}^{2}\left(\frac{\lambda_{1}-\lambda_{2}}{\lambda_{1}+\lambda_{2}-2 \lambda_{3}}\right)^{2}}}{8} .
\end{array}
$$

Here, $g_{Y}$ and $g_{2}$ are the gauge couplings for $\mathrm{U}(1)_{Y}$ and $\mathrm{SU}(2)_{L}$, respectively. The group volume is $\mathcal{V}_{G}=2 \pi^{2}$ for cases (a), (b) and (c.1), and $\mathcal{V}_{G}=4 \pi^{3}$ for case (c.2).

Notice that we can determine whether a solution, $\phi$, is a minimum or not from the sign of $\kappa_{i}-\lambda_{\phi}$. Let $\chi_{i}$ be a scalar orthogonal to $\phi$. Then, its potential can be written as

$$
V\left(\chi_{i}\right)=\frac{\lambda_{\phi}}{4}\left(\phi^{2}+\chi_{i}^{2}\right)^{2}+\frac{\delta}{2} \phi^{2} \chi_{i}^{2}+\ldots,
$$

where $\delta$ breaks the rotational symmetry of $\left(\phi, \chi_{i}\right)$. Since $\kappa_{i}$ can be read off from the mass term for $\chi_{i}$, we get

$$
\kappa_{i}=\lambda_{\phi}+\delta
$$

Thus, for $\phi$ to be a minimum of the action, we need $\kappa_{i}-\lambda_{\phi}>0$ for all $i$.

For case (c.1), we have $\kappa_{3}=\lambda_{\phi}$ and thus there appears a zero mode, which is due to the spontaneous breaking of the PQ symmetry. Its treatment is discussed in appendix E of [41] and we replace

$$
\left.\left.n_{3}^{(\sigma)}\left[\ln \mathcal{A}^{(\sigma)}\right]_{\overline{\mathrm{MS}}}\right|_{\kappa \rightarrow \kappa_{3}, \lambda \rightarrow \lambda_{\phi}} \rightarrow \mathcal{V}_{\sigma} n_{3}^{(\sigma)}\left[\ln \mathcal{A}^{\prime\left(A_{\mu}, \varphi\right)}\right]_{\overline{\mathrm{MS}}}\right|_{g^{2} \rightarrow 0, \lambda \rightarrow \lambda_{\phi}},
$$

with $\mathcal{V}_{\sigma}=2 \pi$

\section{Matching conditions}

This appendix is devoted to the explanation of the one-loop matching conditions for the dimensionless coupling constants. The matching scale is taken to be $\mu_{t}=m_{t}$. For a detailed discussion of the renormalization scheme, see [77, 78]. We assume that $\tan \beta$ and $\cos (\beta-\alpha)$ are renormalized with the $\overline{\mathrm{MS}}$ scheme. In the calculation of one-loop threshold corrections, we utilize the public codes of SARAH [60, 61], FeynArts [62], FeynCalc [63, 64].

\section{D.1 Gauge couplings}

We first evaluate the electric charge at $\mu_{t}$ as

$$
\frac{\left[e^{\mathrm{SM}(5)}\left(\mu_{t}\right)\right]^{2}}{4 \pi}=\frac{\alpha^{\mathrm{SM}(5)}\left(m_{Z}\right)}{1-\frac{b_{e}^{\mathrm{SM}(5)}}{2 \pi} \alpha^{\mathrm{SM}(5)}\left(m_{Z}\right) \ln \frac{\mu_{t}}{m_{Z}}},
$$


where [54]

$$
\begin{aligned}
{\left[\alpha^{\operatorname{SM}(5)}\left(m_{Z}\right)\right]^{-1} } & =127.955 \pm 0.010 \\
b_{e}^{\operatorname{SM}(5)} & =\frac{38}{9} .
\end{aligned}
$$

It is then matched to the THDM electric charge as

$$
e\left(\mu_{t}\right)=\frac{e^{\mathrm{SM}(5)}\left(\mu_{t}\right)}{1-\Delta e},
$$

where

$$
\Delta e=-\frac{\left[e^{\mathrm{SM}(5)}\left(\mu_{t}\right)\right]^{2}}{16 \pi^{2}}\left(-7 \ln \frac{m_{W}}{\mu_{t}}+\frac{1}{3}+\frac{16}{9} \ln \frac{m_{t}}{\mu_{t}}+\frac{1}{3} \ln \frac{m_{H^{+}}}{\mu_{t}}\right)
$$

Next, we calculate the $\overline{\mathrm{MS}}$ masses of the gauge bosons as

$$
m_{V}^{2}\left(\mu_{t}\right)=m_{V}^{2, \mathrm{OS}}+\bar{\Sigma}_{V}^{T}\left(m_{V}^{2}\right)
$$

with $V=W, Z$. Here, $\bar{\Sigma}_{V}^{T}\left(p^{2}\right)$ is the self energy for the transverse mode with $1 / \bar{\varepsilon}$ being subtracted. Here,

$$
\frac{1}{\bar{\varepsilon}}=\frac{2}{4-D}-\gamma_{E}+\ln 4 \pi,
$$

where $D$ is the spacetime dimension and $\gamma_{E}$ is the Euler number. The superscript, OS, indicates the on-shell mass.

Using these, the Weinberg angle is calculated as

$$
\cos \theta_{W}\left(\mu_{t}\right)=\frac{m_{W}\left(\mu_{t}\right)}{m_{Z}\left(\mu_{t}\right)} .
$$

Then, the $\overline{\mathrm{MS}}$ gauge couplings are given by

$$
\begin{aligned}
g_{Y}\left(\mu_{t}\right) & =\frac{e\left(\mu_{t}\right)}{\cos \theta_{W}\left(\mu_{t}\right)}, \\
g_{2}\left(\mu_{t}\right) & =\frac{e\left(\mu_{t}\right)}{\sin \theta_{W}\left(\mu_{t}\right)} .
\end{aligned}
$$

Finally, the strong coupling constant is evaluated with RunDec [65, 66]. Notice that there are no one-loop threshold corrections from the additional Higgs bosons.

For the later convenience, let us define

$$
v\left(\mu_{t}\right)=\frac{2 \sin \theta_{W}\left(\mu_{t}\right)}{e\left(\mu_{t}\right)} m_{W}\left(\mu_{t}\right)
$$

\section{D.2 Yukawa couplings}

The $\overline{\mathrm{MS}}$ tau mass is obtained from

$$
m_{\tau}\left(\mu_{t}\right)=m_{\tau}^{\mathrm{OS}}\left[1+\bar{\Sigma}_{\tau}^{S}\left(m_{\tau}^{2}\right)+\frac{1}{2} \bar{\Sigma}_{\tau}^{L}\left(m_{\tau}^{2}\right)+\frac{1}{2} \bar{\Sigma}_{\tau}^{R}\left(m_{\tau}^{2}\right)\right],
$$


where $\bar{\Sigma}_{\tau}^{S}\left(p^{2}\right), \bar{\Sigma}_{\tau}^{L}\left(p^{2}\right)$ and $\bar{\Sigma}_{\tau}^{R}\left(p^{2}\right)$ are the scalar, the left-handed and the right-handed parts of the self energy with $1 / \bar{\varepsilon}$ being subtracted.

As for the $\overline{\mathrm{MS}}$ masses of the top quark and the bottom quark, we include the four-loop QCD corrections by using RunDec [65, 66]. Then, we add the non-QCD one-loop threshold corrections to the output of RunDec as

$$
m_{f}\left(\mu_{t}\right)=m_{f}^{\mathrm{RunDec}}\left(m_{t}\right)\left[1+\bar{\Sigma}_{f, g_{3}=0}^{S}\left(m_{f}^{2}\right)+\frac{1}{2} \bar{\Sigma}_{f, g_{3}=0}^{L}\left(m_{f}^{2}\right)+\frac{1}{2} \bar{\Sigma}_{f, g_{3}=0}^{R}\left(m_{f}^{2}\right)\right]
$$

for $f=t, b$. Here, $m_{f}^{\text {RunDec }}\left(m_{t}\right)$ is the output of RunDec and the subscript $g_{3}=0$ indicates that the strong coupling is switched off in the calculation.

Then, the $\overline{\mathrm{MS}}$ Yukawa couplings are given by

$$
\begin{aligned}
& y_{t}\left(\mu_{t}\right)=\frac{\sqrt{2}}{\sin \beta\left(\mu_{t}\right)} \frac{m_{t}\left(\mu_{t}\right)}{v\left(\mu_{t}\right)}, \\
& y_{b}\left(\mu_{t}\right)=\frac{\sqrt{2}}{\cos \beta\left(\mu_{t}\right)} \frac{m_{b}\left(\mu_{t}\right)}{v\left(\mu_{t}\right)}, \\
& y_{\tau}\left(\mu_{t}\right)=\frac{\sqrt{2}}{\cos \beta\left(\mu_{t}\right)} \frac{m_{\tau}\left(\mu_{t}\right)}{v\left(\mu_{t}\right)} .
\end{aligned}
$$

\section{D.3 Higgs quartic couplings}

To adjust the Higgs VEVs order by order in perturbative expansions, we extend the scalar potential with tadpole terms as

$$
V_{\mathrm{THDM}} \rightarrow V_{\mathrm{THDM}}+T_{h} h+T_{H} H,
$$

where $T_{h}$ and $T_{H}$ are zero at the tree level.

The $\overline{\mathrm{MS}}$ values of these couplings are chosen as

$$
T_{X}\left(\mu_{t}\right)=\bar{\Gamma}_{X}^{(\operatorname{tad})}
$$

with $X=h, H$. Here, $\bar{\Gamma}_{X}^{(\text {tad })}$ is the tadpole contributions to the effective action with $1 / \bar{\varepsilon}$ being subtracted.

As for the scalars, the $\overline{\mathrm{MS}}$ masses are given by

$$
m_{X}^{2}\left(\mu_{t}\right)=m_{X}^{2, \mathrm{OS}}+\bar{\Sigma}\left(m_{X}^{2}\right),
$$

with $X=h, H, A, H^{+}$. Here, $\bar{\Sigma}_{X}\left(p^{2}\right)$ is the self energy with $1 / \bar{\varepsilon}$ being subtracted. 
The $\overline{\mathrm{MS}}$ Higgs quartic couplings are then obtained as

$$
\begin{aligned}
\lambda_{1}= & \frac{1}{2 v^{2} \cos ^{2} \beta}\left[m_{h}^{2}+m_{H}^{2}-(1-\cos 2 \beta) m_{A}^{2}+\left(m_{H}^{2}-m_{h}^{2}\right) \cos 2 \alpha\right] \\
& +\frac{1}{2 v^{3} \cos \beta}\left[(3 \cos \alpha-\cos (\alpha-2 \beta)) T_{H}-(3 \sin \alpha-\sin (\alpha-2 \beta)) T_{h}\right], \\
\lambda_{2}= & \frac{1}{2 v^{2} \sin ^{2} \beta}\left[m_{h}^{2}+m_{H}^{2}-(1+\cos 2 \beta) m_{A}^{2}-\left(m_{H}^{2}-m_{h}^{2}\right) \cos 2 \alpha\right] \\
& +\frac{1}{2 v^{3} \sin \beta}\left[(3 \sin \alpha+\sin (\alpha-2 \beta)) T_{H}+(3 \cos \alpha+\cos (\alpha-2 \beta)) T_{h}\right], \\
\lambda_{3}= & \frac{1}{v^{2}}\left[2 m_{H^{+}}^{2}-m_{A}^{2}+\left(m_{H}^{2}-m_{h}^{2}\right) \frac{\sin 2 \alpha}{\sin 2 \beta}\right]+\frac{1}{2 v^{3} \sin 2 \beta} \\
& \quad \times\left[(3 \sin (\alpha+\beta)+\sin (\alpha-3 \beta)) T_{H}+(3 \cos (\alpha+\beta)+\cos (\alpha-3 \beta)) T_{h}\right], \\
\lambda_{4}= & \frac{2}{v^{2}}\left(m_{A}^{2}-m_{H^{+}}^{2}\right),
\end{aligned}
$$

where all the quantities appearing in the right-hand side are the $\overline{\mathrm{MS}}$ values; we suppressed the renormalization scale, $\mu_{t}$, for visibility.

\section{E Generation of data points}

In our analysis, we need to generate data points that consist of $\left(m_{H}, m_{A}, \tan \beta, \cos (\beta-\alpha)\right)$ for a fixed $m_{H^{+}}$. We first take a random $\tan \beta$, which is uniformly distributed in the ranges defined in eqs. (5.1)-(5.3). The other variables are generated with the procedure described in this section. The generated data points are then filtered by the perturbative unitarity conditions and are passed to the next analysis. In this appendix, we answer the following questions: (i) what is the appropriate range for $m_{H}, m_{A}$ and $\cos (\beta-\alpha)$ that covers all the points allowed by the perturbative unitarity? (ii) how can we effectively generate data points that are allowed by the perturbative unitarity?

A naive answer to question (i) is that $\left|m_{H^{+}}^{2}-m_{H, A}^{2}\right| \lesssim 8 \pi v^{2}$ and $|\cos (\beta-\alpha)| \leq 1$, where $v \simeq 246 \mathrm{GeV}$. However, they are too weak to be used for the parameter scan. As we can see from figure 4 , the allowed mass differences are smaller than about $200 \mathrm{GeV}$. However, one realizes that $\sqrt{8 \pi} v \simeq 1.2 \mathrm{TeV}$. It also means that $H$ can be as light as $h$ and the mixing angle can become large, which is why we naively expect no constraint on the mixing angle. However, the allowed $|\cos (\beta-\alpha)|$ is smaller than about 0.02 and becomes much smaller in the large $\tan \beta$ regime. Thus, if we scattered the data points over this naive range, we could get only a very few points that satisfy the low energy constraints. That is why we have question (ii).

\section{E.1 Necessary conditions for perturbative unitarity}

Let us first analyze the perturbative unitarity conditions. For arbitrary real numbers $A, B$ and $C$, the inequality,

$$
\left|A \pm \sqrt{B^{2}+C^{2}}\right|<1
$$


can be reduced to

$$
|C|<\sqrt{(|A|-1)^{2}-B^{2}} \&|A|<1 \&|B|<1-|A| .
$$

Applying it to the perturbative unitarity constraints, we get constraints on $\lambda_{1}$ and $\lambda_{2}$ as

$$
\begin{aligned}
\left|\lambda_{1}\right| & <\mathcal{T}, \\
\left|\lambda_{2}\right| & <\mathcal{T}, \\
\left|\lambda_{1}+\lambda_{2}\right| & <\frac{2}{3} \mathcal{T}, \\
\left|\lambda_{1}+\lambda_{2}\right| & <\mathcal{T}+\frac{\lambda_{1} \lambda_{2}}{\mathcal{T}}, \\
\left|\lambda_{1}+\lambda_{2}\right| & <\frac{\mathcal{T}}{3}+\frac{3 \lambda_{1} \lambda_{2}}{\mathcal{T}},
\end{aligned}
$$

which can be reduced to

$$
\begin{aligned}
& \left|\lambda_{1}\right|<\frac{\mathcal{T}}{3}, \\
& \left|\lambda_{2}\right|<\frac{\mathcal{T}}{3} .
\end{aligned}
$$

Here, $\mathcal{T}=8 \pi$. As for $\lambda_{3}$ and $\lambda_{4}$, the constraints have the form of

$$
a_{i} \lambda_{3}+b_{i} \lambda_{4}<c_{i} .
$$

Here, $a_{i}$ 's and $b_{i}$ 's are constants and $c_{i}$ 's are functions of $\lambda_{1}$ and $\lambda_{2}$.

The range of $\lambda_{3}$ and $\lambda_{4}$ satisfying eq. (E.10) can be determined by the simplex method of linear programming. We consider simultaneous equations given by

$$
a_{i} \lambda_{3}+b_{i} \lambda_{4}+z_{i}=c_{i},
$$

where $z_{i}$ 's are the slack variables. Then, we solve them under the constraint of $z_{k}=z_{l}=0$ for each pair of $(k, l)$. The solutions satisfying $z_{i} \geq 0$ correspond to the corners of the allowed region. We search for such solutions and get

$$
\begin{aligned}
\left|\lambda_{3}\right| & <\frac{\mathcal{T}+\sqrt{\mathcal{T}^{2}-3 \mathcal{T}\left|\lambda_{1}+\lambda_{2}\right|+9 \lambda_{1} \lambda_{2}}}{3} \leq \frac{2}{3} \mathcal{T}, \\
\left|\lambda_{4}\right| & <\frac{2}{3} \mathcal{T} \\
\left|\lambda_{3}+\lambda_{4}\right| & <\frac{\mathcal{T}+\sqrt{\mathcal{T}^{2}-3 \mathcal{T}\left|\lambda_{1}+\lambda_{2}\right|+9 \lambda_{1} \lambda_{2}}}{3} \leq \frac{2}{3} \mathcal{T}, \\
\left|2 \lambda_{3}+\lambda_{4}\right| & <\sqrt{\mathcal{T}^{2}-3 \mathcal{T}\left|\lambda_{1}+\lambda_{2}\right|+9 \lambda_{1} \lambda_{2}} \leq \mathcal{T} .
\end{aligned}
$$

\section{E.2 Data generation}

Let us go back to the problem of data generation. We define

$$
\begin{aligned}
& \lambda_{a}=\lambda_{1} \cos ^{2} \beta-\lambda_{2} \sin ^{2} \beta-\left(\lambda_{3}+\lambda_{4}\right) \cos 2 \beta, \\
& \lambda_{b}=\lambda_{1} \cos ^{2} \beta+\lambda_{2} \sin ^{2} \beta+\frac{\lambda_{4}}{2}, \\
& \lambda_{c}=\lambda_{1} \cos ^{2} \beta-\lambda_{2} \sin ^{2} \beta+\lambda_{3} \cos 2 \beta .
\end{aligned}
$$

We will scatter $\left(\lambda_{a}, \lambda_{b}, \lambda_{4}\right)$ instead of $\left(m_{H}, m_{A}, \cos (\beta-\alpha)\right)$. 
We first generate random $\left(\lambda_{a}, \lambda_{b}\right)$. The scattering range is given by

$$
\begin{aligned}
\left|\lambda_{a}\right| & <\frac{\mathcal{T}}{3}(1+2|\cos 2 \beta|), \\
\left|\lambda_{b}\right| & <\frac{2}{3} \mathcal{T}
\end{aligned}
$$

which are derived from the inequalities in the previous subsection. We can further constrain the range with

$$
m_{H^{+}}^{2}-m_{h}^{2}+\lambda_{b} v^{2}=m_{H}^{2}>0 .
$$

Then, we calculate

$$
\begin{aligned}
\sin 2(\beta-\alpha)= & \frac{\lambda_{a} v^{2} \sin 2 \beta}{m_{H^{+}}^{2}-2 m_{h}^{2}+\lambda_{b} v^{2}}, \\
\lambda_{c}= & \frac{2 \cos 2 \beta}{v^{2}}\left[m_{H^{+}}^{2}+\cos 2(\beta-\alpha)\left(m_{H^{+}}^{2}-2 m_{h}^{2}+\lambda_{b} v^{2}\right)\right] \\
& -\lambda_{a} \cos 4 \beta,
\end{aligned}
$$

with the assumption of $\cos 2(\beta-\alpha)<0,{ }^{15}$ and check

$$
\begin{aligned}
|\sin 2(\beta-\alpha)| & <1 \\
\left|\lambda_{c}\right| & <\frac{\mathcal{T}}{3}(1+2|\cos 2 \beta|), \\
\left|\lambda_{a}-\lambda_{c}\right| & <\mathcal{T}|\cos 2 \beta| .
\end{aligned}
$$

If any of them are not satisfied, we step back and regenerate $\left(\lambda_{a}, \lambda_{b}\right)$.

Next, we generate a random $\lambda_{4}$. The scattering range is given by

$$
\begin{aligned}
\left|\lambda_{4}\right| & <\frac{2}{3} \mathcal{T}, \\
m_{H^{+}}^{2}+\frac{\lambda_{4}}{2} v^{2} & =m_{A}^{2}>0, \\
\left|\frac{\lambda_{a}+2 \lambda_{b}+\lambda_{c}}{2 \sin ^{2} \beta}-\lambda_{4}\right| & =\left|2 \lambda_{1} \cot ^{2} \beta\right|<\frac{2}{3} \mathcal{T} \cot ^{2} \beta .
\end{aligned}
$$

If all values of $\lambda_{4}$ have already been excluded, we go back and regenerate $\left(\lambda_{a}, \lambda_{b}\right)$.

Finally, we calculate $m_{H}$ and $m_{A}$ using eqs. (E.21) and (E.28), and $\cos (\beta-\alpha)$ from $\sin 2(\beta-\alpha)$. Notice that we have assumed $\cos 2(\beta-\alpha)<0$ and $\sin (\beta-\alpha)>0$ in this analysis. Then, we output $\left(m_{H}, m_{A}, \tan \beta, \cos (\beta-\alpha)\right)$.

We find that the speed of the data generation is fast enough and $50 \%-60 \%$ of the generated data points satisfy the perturbative unitarity conditions.

\footnotetext{
${ }^{15}$ We could not find any allowed points for the opposite case.
} 


\begin{tabular}{|c|cccccc|}
\hline \multicolumn{7}{|c|}{ Neutral Higgs Couplings } \\
\hline & $g_{X U U}$ & $g_{X D D}$ & $g_{X L L}$ & $g_{X V V}$ & $g_{X A Z}$ & $g_{X H^{ \pm} W^{\mp}}$ \\
\hline$h$ & $\frac{\cos \alpha}{\sin \beta}$ & $-\frac{\sin \alpha}{\cos \beta}$ & $-\frac{\sin \alpha}{\cos \beta}$ & $\sin (\beta-\alpha)$ & $\cos (\beta-\alpha)$ & $\mp \cos (\beta-\alpha)$ \\
$H$ & $\frac{\sin \alpha}{\sin \beta}$ & $\frac{\cos \alpha}{\cos \beta}$ & $\frac{\cos \alpha}{\cos \beta}$ & $\cos (\beta-\alpha)$ & $-\sin (\beta-\alpha)$ & $\pm \sin (\beta-\alpha)$ \\
$A$ & $\cot \beta$ & $\tan \beta$ & $\tan \beta$ & 0 & 0 & 1 \\
\hline
\end{tabular}

Table 5. The SM-value normalized couplings of the neutral Higgs bosons at the tree level. The uptype quarks, the down-type quarks, the leptons, the vector bosons and the neutral Higgs bosons are represented by $U, D, L, V$ and $X$, respectively. The couplings of $A$ to fermions are the pseudo-scalar type and the others are the scalar type.

\section{F Couplings and partial decay widths of heavy higgs bosons}

In this appendix, we summarize couplings and the partial decay widths of the Higgs bosons, which are used for the inputs of HiggsBounds [55-59]. We use the results of [79-81]. The tree level couplings of the neutral Higgs bosons are shown in table 5. They are normalized by the corresponding couplings of the SM Higgs boson having the same mass as that of the decaying particle.

In the following, we use the running mass for the quark mass;

$$
m_{q} \equiv\left(\frac{m_{X}}{\mu_{0}}\right)^{-\frac{2 \alpha_{s}\left(\mu_{0}\right)}{\pi}} m_{q}\left(\mu_{0}\right) \text {, }
$$

where $X$ represents the decaying particle. The reference value $m_{q}\left(\mu_{0}\right)$ is calculated with RunDec $[65,66]$ with $\mu_{0}=500 \mathrm{GeV}$. We define the following variables;

$$
\begin{aligned}
x_{i}^{X} & =\frac{4 m_{i}^{2}}{m_{X}^{2}}, y_{i}=\frac{4 m_{i}^{2}}{m_{Z}^{2}}, \\
\lambda\left(m_{i}^{2}, m_{j}^{2} ; m_{k}^{2}\right) & =\left(1-\frac{m_{i}^{2}}{m_{k}^{2}}-\frac{m_{j}^{2}}{m_{k}^{2}}\right)^{2}-\frac{4 m_{i}^{2} m_{j}^{2}}{m_{k}^{4}} .
\end{aligned}
$$

The loop induced couplings are given by

$$
\begin{aligned}
g_{X g g} & =\left|\frac{\sum_{f=t, b} g_{X f f} A_{1 / 2}^{X}\left(x_{f}^{X}\right)}{A_{1 / 2}^{h}\left(x_{t}^{X}\right)}\right| \\
g_{X \gamma \gamma} & =\left|\frac{\sum_{f=t, b, \tau} N_{c}^{f} Q_{f}^{2} g_{X f f} A_{1 / 2}^{X}\left(x_{f}^{X}\right)+g_{X W W} A_{1}^{X}\left(x_{W}^{X}\right)+g_{X H^{+} H^{-}} A_{0}^{X}\left(x_{H^{+}}^{X}\right)}{\frac{4}{3} A_{1 / 2}^{h}\left(x_{t}^{X}\right)+A_{1}^{h}\left(x_{W}^{X}\right)}\right|, \\
g_{X Z \gamma} & =\left|\frac{\sum_{f=t, b, \tau} g_{X f f} \bar{A}_{f}^{X}\left(x_{f}^{X}, y_{f}\right)+g_{X W W} \bar{A}_{W}^{X}\left(x_{W}^{X}, y_{W}\right)+g_{X H^{+} H^{-}} \bar{A}_{H^{ \pm}}\left(x_{H^{+}}^{X}, y_{H^{+}}\right)}{\bar{A}_{t}^{h}\left(x_{t}^{X}, y_{t}\right)+\bar{A}_{W}^{h}\left(x_{W}^{X}, y_{W}\right)}\right|,
\end{aligned}
$$


where

$$
\begin{aligned}
A_{0}^{h, H} & =-x[1-x f(x)], \\
A_{1 / 2}^{h, H} & =2 x[1+(1-x) f(x)], \\
A_{1}^{h, H} & =-[2+3 x+3 x(2-x) f(x)], \\
A_{1 / 2}^{A} & =2 x f(x),
\end{aligned}
$$

and

$$
\begin{aligned}
\bar{A}_{H^{+}}^{h, H}(x, y) & =\frac{\cos 2 \theta_{W}}{\cos \theta_{W}} I_{1}(x, y), \\
\bar{A}_{f}^{h, H}(x, y) & =2 N_{c}^{f} \frac{Q_{f}\left(I_{3}^{f}-2 Q_{f} \sin ^{2} \theta_{W}\right)}{\cos \theta_{W}}\left[I_{1}(x, y)-I_{2}(x, y)\right], \\
\bar{A}_{W}^{h, H}(x, y) & \left.=\cos \theta_{W}\left\{\begin{array}{l}
4\left(3-\tan ^{2} \theta_{W}\right) I_{2}(x, y) \\
+
\end{array}\right]\left[\left(1+\frac{2}{x}\right) \tan ^{2} \theta_{W}-\left(5+\frac{2}{x}\right) I_{1}(x, y)\right]\right\}, \\
\bar{A}_{f}^{A}(x, y) & =2 N_{c}^{f} \frac{Q_{f}\left(I_{3}^{f}-2 Q_{f} \sin ^{2} \theta_{W}\right)}{\cos \theta_{W}} I_{2}(x, y) .
\end{aligned}
$$

Here, the tri-linear Higgs couplings are given by

$$
\begin{aligned}
g_{h H^{+} H^{-}} & =\frac{\left(m_{h}^{2}-2 m_{H^{+}}^{2}\right) \cos (\alpha-3 \beta)+\left(2 m_{H^{+}}^{2}+3 m_{h}^{2}-4 m_{A}^{2}\right) \cos (\alpha+\beta)}{4 m_{H^{+}}^{2} \sin 2 \beta}, \\
g_{H H^{+} H^{-}} & =\frac{\left(m_{H}^{2}-2 m_{H^{+}}^{2}\right) \sin (\alpha-3 \beta)+\left(2 m_{H^{+}}^{2}+3 m_{H}^{2}-4 m_{A}^{2}\right) \sin (\alpha+\beta)}{4 m_{H^{+}}^{2} \sin 2 \beta} .
\end{aligned}
$$

Notice that $g_{A H^{+} H^{-}}=0$. The functions used in the above equations are defined as

$$
\begin{aligned}
& I_{1}(x, y)=\frac{x y}{2(x-y)}+\frac{x^{2} y^{2}}{2(x-y)^{2}}[f(x)-f(y)]+\frac{x^{2} y}{(x-y)^{2}}[g(x)-g(y)], \\
& I_{2}(x, y)=-\frac{x y}{2(x-y)}[f(x)-f(y)],
\end{aligned}
$$

and

$$
\begin{aligned}
& f(x)= \begin{cases}\arcsin ^{2} \frac{1}{\sqrt{x}} & x \geq 1 \\
-\frac{1}{4}\left[\log \frac{1+\sqrt{1-x}}{1-\sqrt{1-x}}-i \pi\right]^{2} & x<1\end{cases} \\
& g(x)=\left\{\begin{array}{ll}
\sqrt{x-1} \arcsin \frac{1}{\sqrt{x}} & x \geq 1 \\
\frac{\sqrt{1-x}}{2}\left[\log \frac{1+\sqrt{1-x}}{1-\sqrt{1-x}}-i \pi\right] & x<1
\end{array} .\right.
\end{aligned}
$$


We include the following non-SM partial decay widths of the neutral Higgs bosons;

$$
\begin{aligned}
\Gamma(H \rightarrow X X) & =\frac{G_{F} m_{Z}^{4}}{16 \sqrt{2} \pi m_{H}} g_{H X X}^{2} \sqrt{1-4 \frac{m_{X}^{2}}{m_{H}^{2}}}, \\
\Gamma\left(X_{i} \rightarrow X_{j} Z\right) & =\frac{G_{F} m_{X_{i}}^{3}}{8 \sqrt{2} \pi} g_{X_{i} X_{j} Z^{\lambda}}^{3 / 2}\left(m_{Z}^{2}, m_{X_{j}}^{2} ; m_{X_{i}}^{2}\right), \\
\Gamma\left(X \rightarrow H^{-} W^{+}\right) & =\Gamma\left(X \rightarrow H^{+} W^{-}\right) \\
& =\frac{G_{F} m_{X}^{3}}{8 \sqrt{2} \pi} g_{X H^{ \pm} W^{2}}^{2} \lambda^{3 / 2}\left(m_{W}^{2}, m_{H^{+}}^{2} ; m_{X}^{2}\right),
\end{aligned}
$$

where the relevant couplings are given by

$$
\begin{aligned}
g_{H h h} & \left.=\frac{\cos (\beta-\alpha)}{m_{Z}^{2} \sin 2 \beta}\left[\left(m_{H}^{2}+2 m_{h}^{2}-3 m_{A}^{2}\right) \sin 2 \alpha+m_{A}^{2} \sin 2 \beta\right)\right], \\
g_{H A A} & \left.=\frac{1}{2 m_{Z}^{2} \sin 2 \beta}\left[\left(m_{H}^{2}-2 m_{A}^{2}\right) \sin (\alpha-3 \beta)+\left(3 m_{H}^{2}-2 m_{A}^{2}\right) \sin (\alpha+\beta)\right)\right] .
\end{aligned}
$$

As for the charged Higgs boson, we consider the following partial decay widths;

$$
\begin{aligned}
\Gamma\left(H^{+} \rightarrow X W^{+}\right) & =\frac{G_{F} m_{H^{+}}^{3}}{8 \sqrt{2} \pi} g_{X H^{-} W^{+}}^{2} \lambda^{3 / 2}\left(m_{W}^{2}, m_{X}^{2} ; m_{H^{+}}^{2}\right), \\
\Gamma\left(H^{+} \rightarrow t b\right) & =\frac{3 G_{F} m_{H^{+}}}{4 \sqrt{2} \pi}\left[m_{t}^{2} \cot ^{2} \beta+m_{b}^{2} \tan ^{2} \beta\right] \lambda^{1 / 2}\left(m_{t}^{2}, m_{b}^{2} ; m_{H^{+}}^{2}\right), \\
\Gamma\left(H^{+} \rightarrow \tau \nu\right) & =\frac{G_{F} m_{H^{+}}}{4 \sqrt{2} \pi} m_{\tau}^{2} \tan ^{2} \beta\left(1-\frac{m_{\tau}^{2}}{m_{H^{+}}^{2}}\right)^{3} .
\end{aligned}
$$

Open Access. This article is distributed under the terms of the Creative Commons Attribution License (CC-BY 4.0), which permits any use, distribution and reproduction in any medium, provided the original author(s) and source are credited.

\section{References}

[1] R.D. Peccei and H.R. Quinn, CP Conservation in the Presence of Instantons, Phys. Rev. Lett. 38 (1977) 1440 [INSPIRE].

[2] S. Weinberg, A New Light Boson?, Phys. Rev. Lett. 40 (1978) 223 [INSPIRE].

[3] F. Wilczek, Problem of Strong $P$ and T Invariance in the Presence of Instantons, Phys. Rev. Lett. 40 (1978) 279 [inSPIRE].

[4] L.F. Abbott and P. Sikivie, A Cosmological Bound on the Invisible Axion, Phys. Lett. 120B (1983) 133 [INSPIRE].

[5] M. Dine and W. Fischler, The Not So Harmless Axion, Phys. Lett. 120B (1983) 137 [INSPIRE].

[6] J. Preskill, M.B. Wise and F. Wilczek, Cosmology of the Invisible Axion, Phys. Lett. 120B (1983) 127 [INSPIRE].

[7] M. Kuster, G. Raffelt and B. Beltran, Axions: Theory, cosmology, and experimental searches. Proceedings, 1st Joint ILIAS-CERN-CAST axion training, Geneva, Switzerland, November 30 - December 2, 2005, Lect. Notes Phys. 741 (2008) 1. 
[8] M.A. Shifman, A.I. Vainshtein and V.I. Zakharov, Can Confinement Ensure Natural CP Invariance of Strong Interactions?, Nucl. Phys. B 166 (1980) 493 [InSPIRE].

[9] J.E. Kim, Weak Interaction Singlet and Strong CP Invariance, Phys. Rev. Lett. 43 (1979) 103 [INSPIRE].

[10] A.R. Zhitnitsky, On Possible Suppression of the Axion Hadron Interactions (in Russian), Sov. J. Nucl. Phys. 31 (1980) 260 [inSPIRE].

[11] M. Dine, W. Fischler and M. Srednicki, A Simple Solution to the Strong CP Problem with a Harmless Axion, Phys. Lett. 104B (1981) 199 [InSPIRE].

[12] W.A. Bardeen, On naturalness in the standard model, in Ontake Summer Institute on Particle Physics Ontake Mountain, Japan, August 27-September 2, 1995, FERMILAB-CONF-95-391 [INSPIRE].

[13] C.T. Hill, Conjecture on the physical implications of the scale anomaly, hep-th/0510177 [INSPIRE].

[14] H. Aoki and S. Iso, Revisiting the Naturalness Problem - Who is afraid of quadratic divergences? -, Phys. Rev. D 86 (2012) 013001 [arXiv: 1201.0857] [INSPIRE].

[15] K. Allison, C.T. Hill and G.G. Ross, An ultra-weak sector, the strong CP problem and the pseudo-Goldstone dilaton, Nucl. Phys. B 891 (2015) 613 [arXiv:1409.4029] [INSPIRE].

[16] K. Allison, C.T. Hill and G.G. Ross, Ultra-weak sector, Higgs boson mass and the dilaton, Phys. Lett. B 738 (2014) 191 [arXiv: 1404.6268] [InSPIRE].

[17] F. Vissani, Do experiments suggest a hierarchy problem?, Phys. Rev. D 57 (1998) 7027 [hep-ph/9709409] [INSPIRE].

[18] M. Farina, D. Pappadopulo and A. Strumia, A modified naturalness principle and its experimental tests, JHEP 08 (2013) 022 [arXiv: 1303.7244] [INSPIRE].

[19] R. Foot, A. Kobakhidze, K.L. McDonald and R.R. Volkas, Poincaré protection for a natural electroweak scale, Phys. Rev. D 89 (2014) 115018 [arXiv:1310.0223] [INSPIRE].

[20] J.D. Clarke, R. Foot and R.R. Volkas, Natural leptogenesis and neutrino masses with two Higgs doublets, Phys. Rev. D 92 (2015) 033006 [arXiv: 1505.05744] [INSPIRE].

[21] V. Branchina, F. Contino and P.M. Ferreira, Electroweak vacuum lifetime in two Higgs doublet models, JHEP 11 (2018) 107 [arXiv:1807.10802] [INSPIRE].

[22] M.E. Krauss, T. Opferkuch and F. Staub, The Ultraviolet Landscape of Two-Higgs Doublet Models, Eur. Phys. J. C 78 (2018) 1020 [arXiv:1807.07581] [InSPIRE].

[23] P. Basler, P.M. Ferreira, M. Mühlleitner and R. Santos, High scale impact in alignment and decoupling in two-Higgs doublet models, Phys. Rev. D 97 (2018) 095024 [arXiv:1710.10410] [INSPIRE].

[24] N. Chakrabarty and B. Mukhopadhyaya, High-scale validity of a two Higgs doublet scenario: predicting collider signals, Phys. Rev. D 96 (2017) 035028 [arXiv:1702.08268] [INSPIRE].

[25] N. Chakrabarty and B. Mukhopadhyaya, High-scale validity of a two Higgs doublet scenario: metastability included, Eur. Phys. J. C 77 (2017) 153 [arXiv:1603.05883] [INSPIRE].

[26] V. Cacchio, D. Chowdhury, O. Eberhardt and C.W. Murphy, Next-to-leading order unitarity fits in Two-Higgs-Doublet models with soft $\mathbb{Z}_{2}$ breaking, JHEP 11 (2016) 026 [arXiv: 1609.01290] [INSPIRE]. 
[27] E. Bagnaschi, F. Brümmer, W. Buchmüller, A. Voigt and G. Weiglein, Vacuum stability and supersymmetry at high scales with two Higgs doublets, JHEP 03 (2016) 158 [arXiv: 1512.07761] [INSPIRE].

[28] D. Chowdhury and O. Eberhardt, Global fits of the two-loop renormalized Two-Higgs-Doublet model with soft $Z_{2}$ breaking, JHEP 11 (2015) 052 [arXiv:1503.08216] [INSPIRE].

[29] P. Ferreira, H.E. Haber and E. Santos, Preserving the validity of the Two-Higgs Doublet Model up to the Planck scale, Phys. Rev. D 92 (2015) 033003 [Erratum ibid. D 94 (2016) 059903] [arXiv: 1505.04001] [INSPIRE].

[30] D. Das and I. Saha, Search for a stable alignment limit in two-Higgs-doublet models, Phys. Rev. D 91 (2015) 095024 [arXiv: 1503.02135] [INSPIRE].

[31] N. Chakrabarty, U.K. Dey and B. Mukhopadhyaya, High-scale validity of a two-Higgs doublet scenario: a study including LHC data, JHEP 12 (2014) 166 [arXiv:1407.2145] [INSPIRE].

[32] B. Grzadkowski, O.M. Ogreid and P. Osland, Diagnosing CP properties of the 2HDM, JHEP 01 (2014) 105 [arXiv: 1309.6229] [inSPIRE].

[33] J. Shu and Y. Zhang, Impact of a CP-violating Higgs Sector: From LHC to Baryogenesis, Phys. Rev. Lett. 111 (2013) 091801 [arXiv:1304.0773] [INSPIRE].

[34] S.R. Coleman, The Fate of the False Vacuum. 1. Semiclassical Theory, Phys. Rev. D 15 (1977) 2929 [Erratum ibid. D 16 (1977) 1248] [inSPIRE].

[35] C.G. Callan Jr. and S.R. Coleman, The Fate of the False Vacuum. 2. First Quantum Corrections, Phys. Rev. D 16 (1977) 1762 [InSPIRE].

[36] M. Endo, T. Moroi, M.M. Nojiri and Y. Shoji, Renormalization-Scale Uncertainty in the Decay Rate of False Vacuum, JHEP 01 (2016) 031 [arXiv:1511.04860] [INSPIRE].

[37] G. Isidori, G. Ridolfi and A. Strumia, On the metastability of the standard model vacuum, Nucl. Phys. B 609 (2001) 387 [hep-ph/0104016] [INSPIRE].

[38] M. Endo, T. Moroi, M.M. Nojiri and Y. Shoji, False Vacuum Decay in Gauge Theory, JHEP 11 (2017) 074 [arXiv:1704.03492] [INSPIRE].

[39] A. Andreassen, W. Frost and M.D. Schwartz, Scale Invariant Instantons and the Complete Lifetime of the Standard Model, Phys. Rev. D 97 (2018) 056006 [arXiv:1707.08124] [INSPIRE].

[40] S. Chigusa, T. Moroi and Y. Shoji, State-of-the-Art Calculation of the Decay Rate of Electroweak Vacuum in the Standard Model, Phys. Rev. Lett. 119 (2017) 211801 [arXiv: 1707.09301] [INSPIRE].

[41] S. Chigusa, T. Moroi and Y. Shoji, Decay Rate of Electroweak Vacuum in the Standard Model and Beyond, Phys. Rev. D 97 (2018) 116012 [arXiv:1803.03902] [InSPIRE].

[42] R.D. Peccei, The strong CP problem and axions, Lect. Notes Phys. 741 (2008) 3 [hep-ph/0607268] [INSPIRE].

[43] M.S. Turner, Windows on the Axion, Phys. Rept. 197 (1990) 67 [INSPIRE].

[44] Planck collaboration, Planck 2018 results. VI. Cosmological parameters, arXiv: 1807.06209 [INSPIRE].

[45] A. Arbey, F. Mahmoudi, O. Stal and T. Stefaniak, Status of the Charged Higgs Boson in Two Higgs Doublet Models, Eur. Phys. J. C 78 (2018) 182 [arXiv:1706.07414] [INSPIRE]. 
[46] M. Misiak and M. Steinhauser, Weak radiative decays of the $B$ meson and bounds on $M_{H^{ \pm}}$in the Two-Higgs-Doublet Model, Eur. Phys. J. C 77 (2017) 201 [arXiv:1702. 04571] [InSPIRE].

[47] J. Haller, A. Hoecker, R. Kogler, K. Mönig, T. Peiffer and J. Stelzer, Update of the global electroweak fit and constraints on two-Higgs-doublet models, Eur. Phys. J. C 78 (2018) 675 [arXiv: 1803.01853] [INSPIRE].

[48] T. Enomoto and R. Watanabe, Flavor constraints on the Two Higgs Doublet Models of $Z_{2}$ symmetric and aligned types, JHEP 05 (2016) 002 [arXiv:1511.05066] [INSPIRE].

[49] S. Kanemura, T. Kubota and E. Takasugi, Lee-Quigg-Thacker bounds for Higgs boson masses in a two doublet model, Phys. Lett. B 313 (1993) 155 [hep-ph/9303263] [INSPIRE].

[50] A.G. Akeroyd, A. Arhrib and E.-M. Naimi, Note on tree level unitarity in the general two Higgs doublet model, Phys. Lett. B 490 (2000) 119 [hep-ph/0006035] [INSPIRE].

[51] W. Grimus, L. Lavoura, O.M. Ogreid and P. Osland, A precision constraint on multi-Higgs-doublet models, J. Phys. G 35 (2008) 075001 [arXiv:0711.4022] [InSPIRE].

[52] W. Grimus, L. Lavoura, O.M. Ogreid and P. Osland, The oblique parameters in multi-Higgs-doublet models, Nucl. Phys. B 801 (2008) 81 [arXiv:0802.4353] [INSPIRE].

[53] D. Eriksson, J. Rathsman and O. Stal, 2HDMC: Two-Higgs-Doublet Model Calculator Physics and Manual, Comput. Phys. Commun. 181 (2010) 189 [arXiv:0902.0851] [INSPIRE].

[54] Particle Data Group collaboration, Review of Particle Physics, Phys. Rev. D 98 (2018) 030001 [INSPIRE].

[55] P. Bechtle et al., HiggsBounds-4: Improved Tests of Extended Higgs Sectors against Exclusion Bounds from LEP, the Tevatron and the LHC, Eur. Phys. J. C 74 (2014) 2693 [arXiv: 1311.0055] [INSPIRE].

[56] P. Bechtle et al., Recent Developments in HiggsBounds and a Preview of HiggsSignals, PoS (CHARGED 2012) 024 [arXiv: 1301.2345] [INSPIRE].

[57] P. Bechtle, O. Brein, S. Heinemeyer, G. Weiglein and K.E. Williams, HiggsBounds 2.0.0: Confronting Neutral and Charged Higgs Sector Predictions with Exclusion Bounds from LEP and the Tevatron, Comput. Phys. Commun. 182 (2011) 2605 [arXiv:1102.1898] [INSPIRE].

[58] P. Bechtle, O. Brein, S. Heinemeyer, G. Weiglein and K.E. Williams, HiggsBounds: Confronting Arbitrary Higgs Sectors with Exclusion Bounds from LEP and the Tevatron, Comput. Phys. Commun. 181 (2010) 138 [arXiv:0811.4169] [InSPIRE].

[59] P. Bechtle, S. Heinemeyer, O. Stal, T. Stefaniak and G. Weiglein, Applying Exclusion Likelihoods from LHC Searches to Extended Higgs Sectors, Eur. Phys. J. C 75 (2015) 421 [arXiv: 1507.06706] [INSPIRE].

[60] F. Staub, T. Ohl, W. Porod and C. Speckner, A Tool Box for Implementing Supersymmetric Models, Comput. Phys. Commun. 183 (2012) 2165 [arXiv:1109.5147] [InSPIRE].

[61] F. Staub, Exploring new models in all detail with SARAH, Adv. High Energy Phys. 2015 (2015) 840780 [arXiv: 1503.04200] [INSPIRE].

[62] T. Hahn, Generating Feynman diagrams and amplitudes with FeynArts 3, Comput. Phys. Commun. 140 (2001) 418 [hep-ph/0012260] [INSPIRE].

[63] R. Mertig, M. Böhm and A. Denner, FEYN CALC: Computer algebraic calculation of Feynman amplitudes, Comput. Phys. Commun. 64 (1991) 345 [INSPIRE]. 
[64] V. Shtabovenko, R. Mertig and F. Orellana, New Developments in FeynCalc 9.0, Comput. Phys. Commun. 207 (2016) 432 [arXiv:1601.01167] [InSPIRE].

[65] K.G. Chetyrkin, J.H. Kuhn and M. Steinhauser, RunDec: A mathematica package for running and decoupling of the strong coupling and quark masses, Comput. Phys. Commun. 133 (2000) 43 [hep-ph/0004189] [INSPIRE].

[66] F. Herren and M. Steinhauser, Version 3 of RunDec and CRunDec, Comput. Phys. Commun. 224 (2018) 333 [arXiv:1703.03751] [INSPIRE].

[67] N.G. Deshpande and E. Ma, Pattern of Symmetry Breaking with Two Higgs Doublets, Phys. Rev. D 18 (1978) 2574 [INSPIRE].

[68] LCC Physics Working Group collaboration, Tests of the Standard Model at the International Linear Collider, arXiv:1908.11299 [INSPIRE].

[69] ATLAS collaboration, Combined measurements of Higgs boson production and decay using up to $80 \mathrm{fb}^{-1}$ of proton-proton collision data at $\sqrt{\mathrm{s}}=13 \mathrm{TeV}$ collected with the ATLAS experiment, Phys. Rev. D 101 (2020) 012002 [arXiv:1909.02845] [INSPIRE].

[70] J. Hardy and I.S. Towner, $\left|V_{u d}\right|$ from nuclear $\beta$ decays, PoS(CKM2016) 028.

[71] HFLAV collaboration, Averages of b-hadron, c-hadron and $\tau$-lepton properties as of 2018, arXiv: 1909.12524 [INSPIRE].

[72] M. Misiak et al., Updated NNLO QCD predictions for the weak radiative B-meson decays, Phys. Rev. Lett. 114 (2015) 221801 [arXiv:1503.01789] [INSPIRE].

[73] M. Czakon, P. Fiedler, T. Huber, M. Misiak, T. Schutzmeier and M. Steinhauser, The $\left(Q_{7}, Q_{1,2}\right)$ contribution to $\bar{B} \rightarrow X_{s} \gamma$ at $\mathcal{O}\left(\alpha_{\mathrm{s}}^{2}\right)$, JHEP 04 (2015) 168 [arXiv:1503.01791] [INSPIRE].

[74] Flavour Lattice Averaging Group collaboration, FLAG Review 2019, Eur. Phys. J. C 80 (2020) 113 [arXiv: 1902.08191] [INSPIRE].

[75] Fermilab Lattice and MILC collaborations, $B_{(s)}^{0}$-mixing matrix elements from lattice QCD for the Standard Model and beyond, Phys. Rev. D 93 (2016) 113016 [arXiv: 1602.03560] [INSPIRE].

[76] S.R. Coleman, V. Glaser and A. Martin, Action Minima Among Solutions to a Class of Euclidean Scalar Field Equations, Commun. Math. Phys. 58 (1978) 211 [InSPIRE].

[77] A. Denner, Techniques for calculation of electroweak radiative corrections at the one loop level and results for W physics at LEP-200, Fortsch. Phys. 41 (1993) 307 [arXiv:0709.1075] [INSPIRE].

[78] L. Altenkamp, S. Dittmaier and H. Rzehak, Renormalization schemes for the Two-Higgs-Doublet Model and applications to $h \rightarrow W W / Z Z \rightarrow 4$ fermions, JHEP 09 (2017) 134 [arXiv: 1704.02645] [INSPIRE].

[79] A. Djouadi, J. Kalinowski and P.M. Zerwas, Two and three-body decay modes of SUSY Higgs particles, Z. Phys. C 70 (1996) 435 [hep-ph/9511342] [INSPIRE].

[80] A. Djouadi, The anatomy of electro-weak symmetry breaking. II. The Higgs bosons in the minimal supersymmetric model, Phys. Rept. 459 (2008) 1 [hep-ph/0503173] [INSPIRE].

[81] M. Spira, Higgs Boson Production and Decay at Hadron Colliders, Prog. Part. Nucl. Phys. 95 (2017) 98 [arXiv:1612.07651] [INSPIRE]. 